Supporting Information for Manuscript

An NGS-independent strategy for proteome-wide identification of single amino acid polymorphisms by mass spectrometry

Yun Xiong ${ }^{1}$, Yufeng Guo', Weidi Xiao ${ }^{2}$, Qichen $\mathrm{Cao}^{1}$, Shanshan $\mathrm{Li}^{1}$, Xianni $\mathrm{Qi}^{1}$, Zhidan Zhang ${ }^{1}$, Qinhong Wang ${ }^{1, *}$, Wenqing Shui ${ }^{1, *}$

${ }^{1}$ Key Laboratory of Systems Microbial Biotechnology, Tianjin Institute of Industrial Biotechnology, Chinese Academy of Sciences, Tianjin 300308, China

${ }^{2}$ College of Life Sciences, Nankai University, Tianjin 300071, China

Content of SI materials

Figure S1. Identification of putative SAP peptides using the error-tolerant search by Mascot or de novo sequencing by PepNovo+.

Figure S2. MSMS spectra of 50 non-mapping SAP peptides.

Figure S3. Sanger sequencing confirms the nsSNP sites translating to SAPs in selected peptides.

Figure S4. MSMS spectra of nine SAP peptides repetitively identified in DDA analysis (left) and PRM analysis (right).

Figure S5. MSMS spectra and extracted ion chromatograms of the SAP peptide QAPAFLGPDGLK detected by PRM analysis in total cell extracts, extracts with low spike-in or high spike-in amounts of the synthetic SAP peptide.

Figure S6. MSMS spectra and extracted ion chromatograms of the SAP peptide IMDQGGLVSDDIMVNK detected by PRM analysis in total cell extracts, extracts with low spike-in or high spike-in amounts of the synthetic SAP peptide.

Figure S7. MSMS spectra and extracted ion chromatograms of the SAP peptide VVITAPSSTAPMFVMGVNEEKK detected by PRM analysis in total cell extracts, extracts with low spike-in or high spike-in amounts of the synthetic SAP peptide.

Table S1. Protein identification summary from regular search or error-tolerant search by ProteinPilot, and PSMs for all putative SAP peptides identified in this study 
Table S2. SAP peptides validated by regular search using three different engines Table S3. SIFT and Polyphen-2 scores of 327 validated SAPs

Table S4. Mass accuracy, Mascot scores and D scores of 22 non-mapping SAP peptides 
A

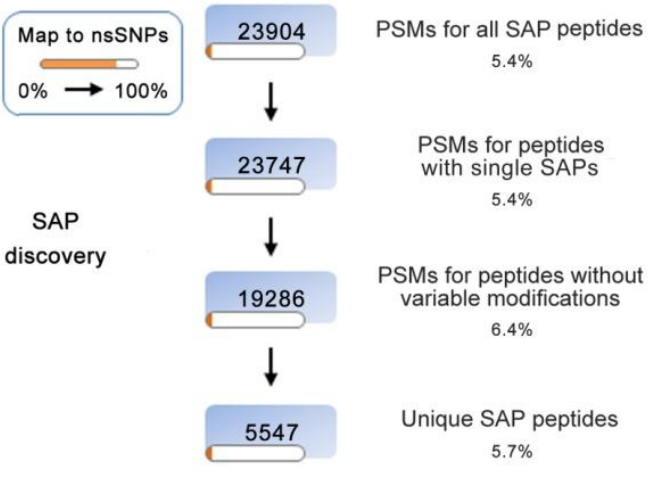

B

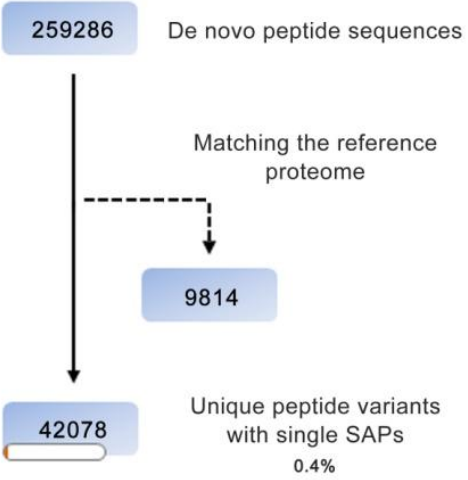

Figure S1. Identification of putative SAP peptides using the error-tolerant search by Mascot (A) and de novo sequencing by PepNovo+ (B). In Mascot search, the proteomic dataset was searched against a reference proteome by error-tolerant search to acquire SAP peptides at a global FDR $<1 \%$. After data filtration, unique SAPs of distinct sequences and no variable modifications were obtained. In PepNovo+ search, peptide sequences assigned de novo were compared with the reference proteome to obtain unique peptide variants with single SAPs. The mapping rate to nsSNPs is annotated at each level. 
Category 1

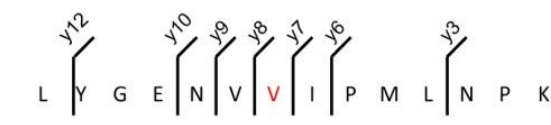
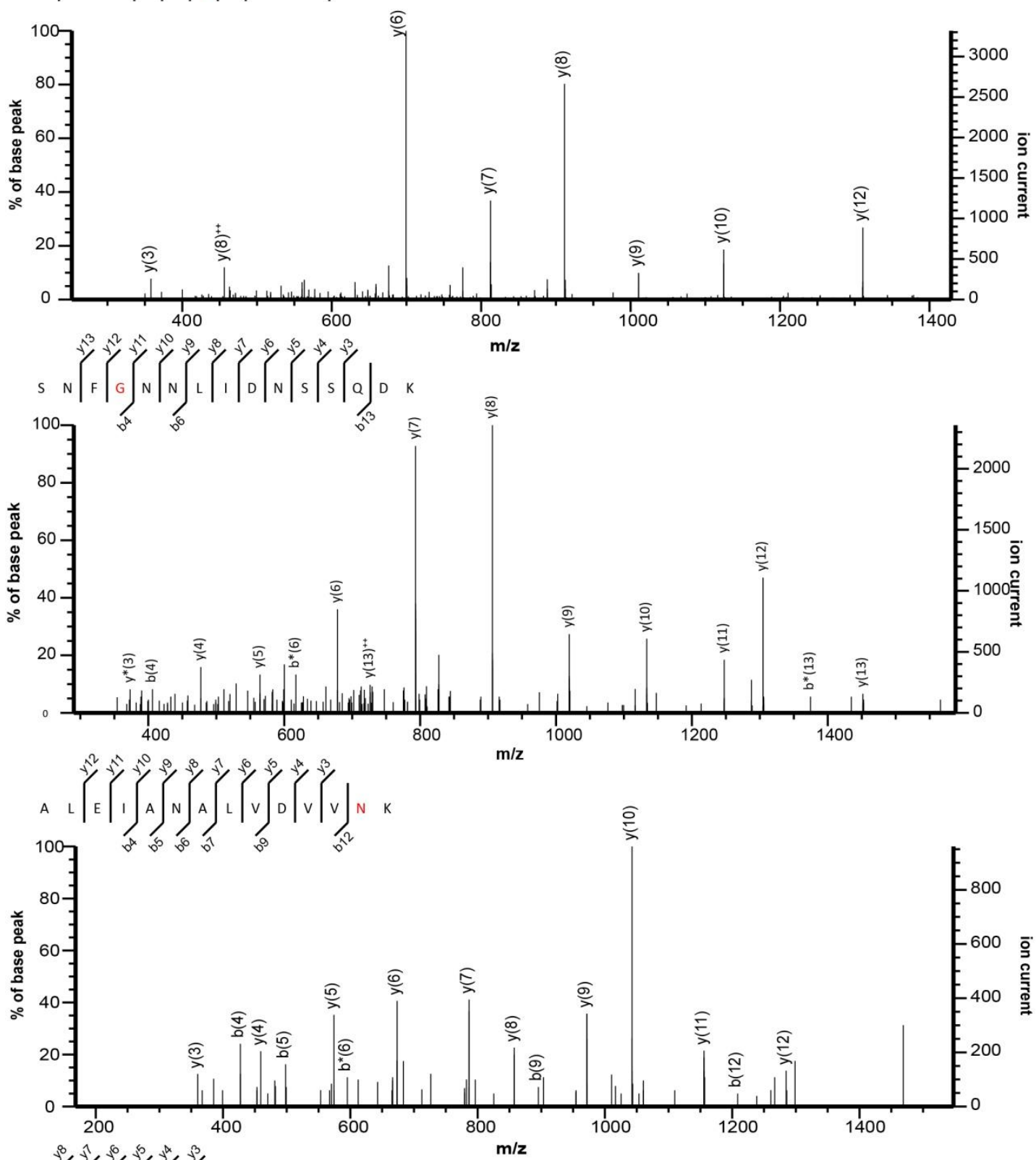

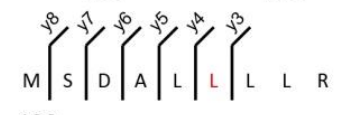

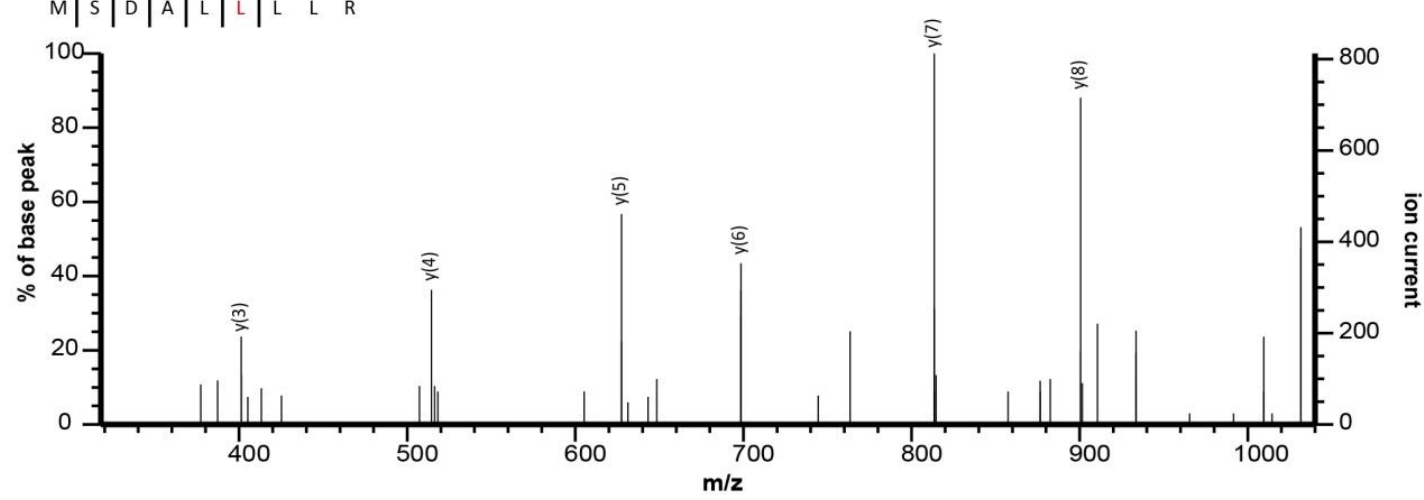

S-4 


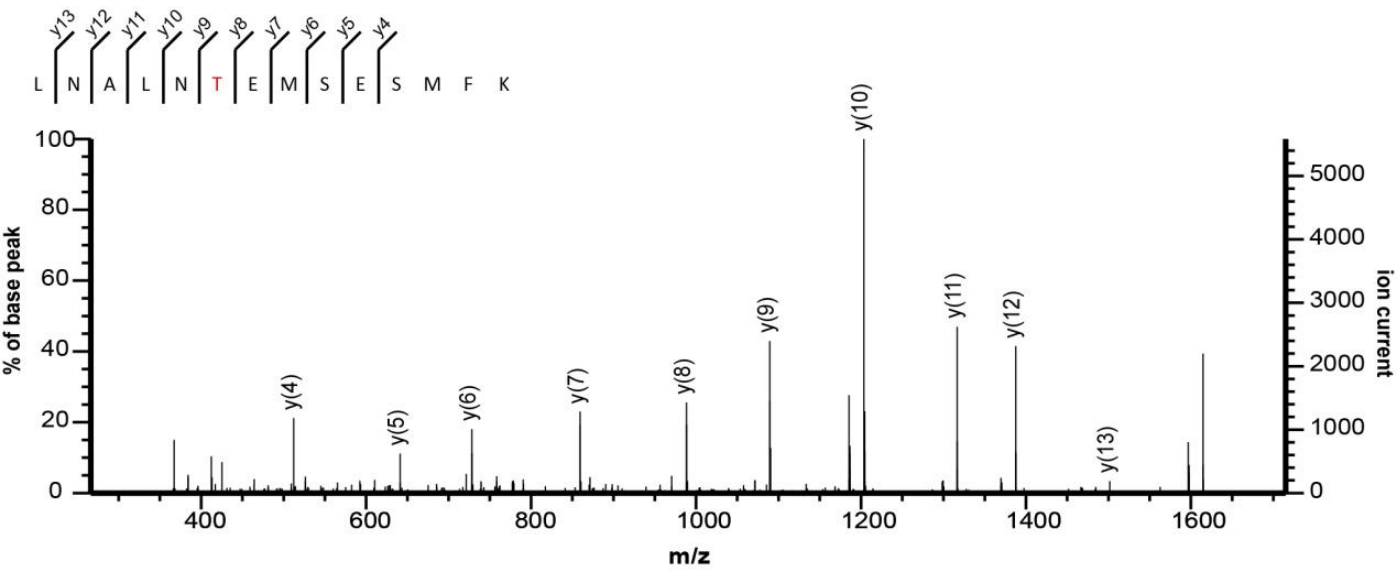

Catrgory 2
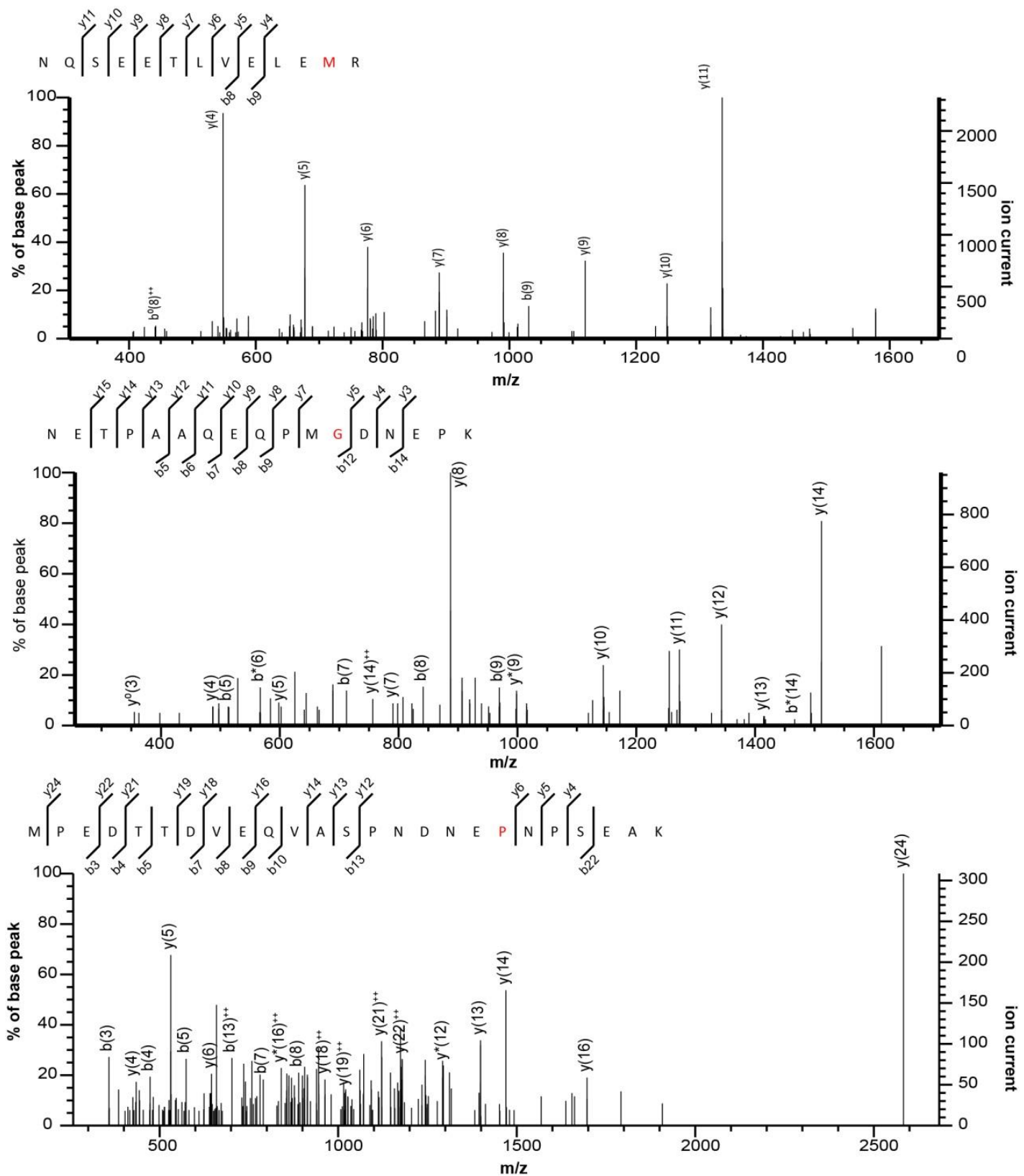

S-5 


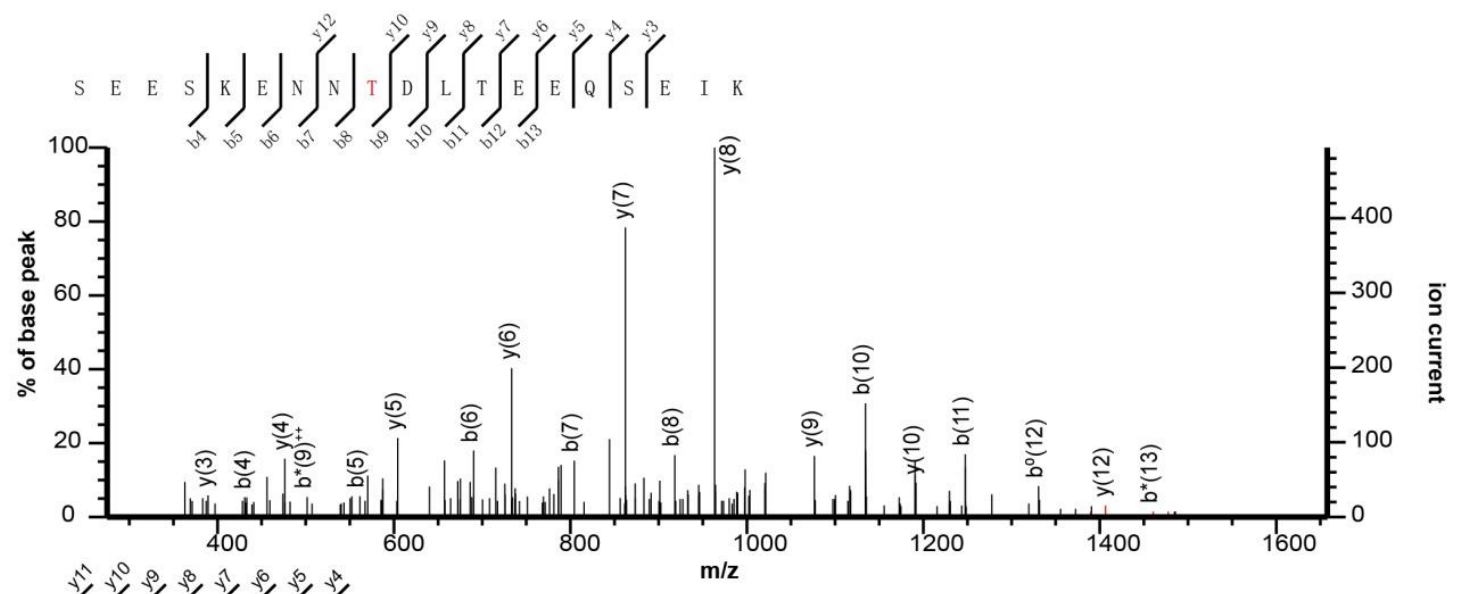

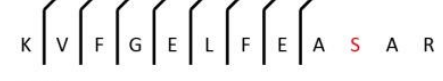
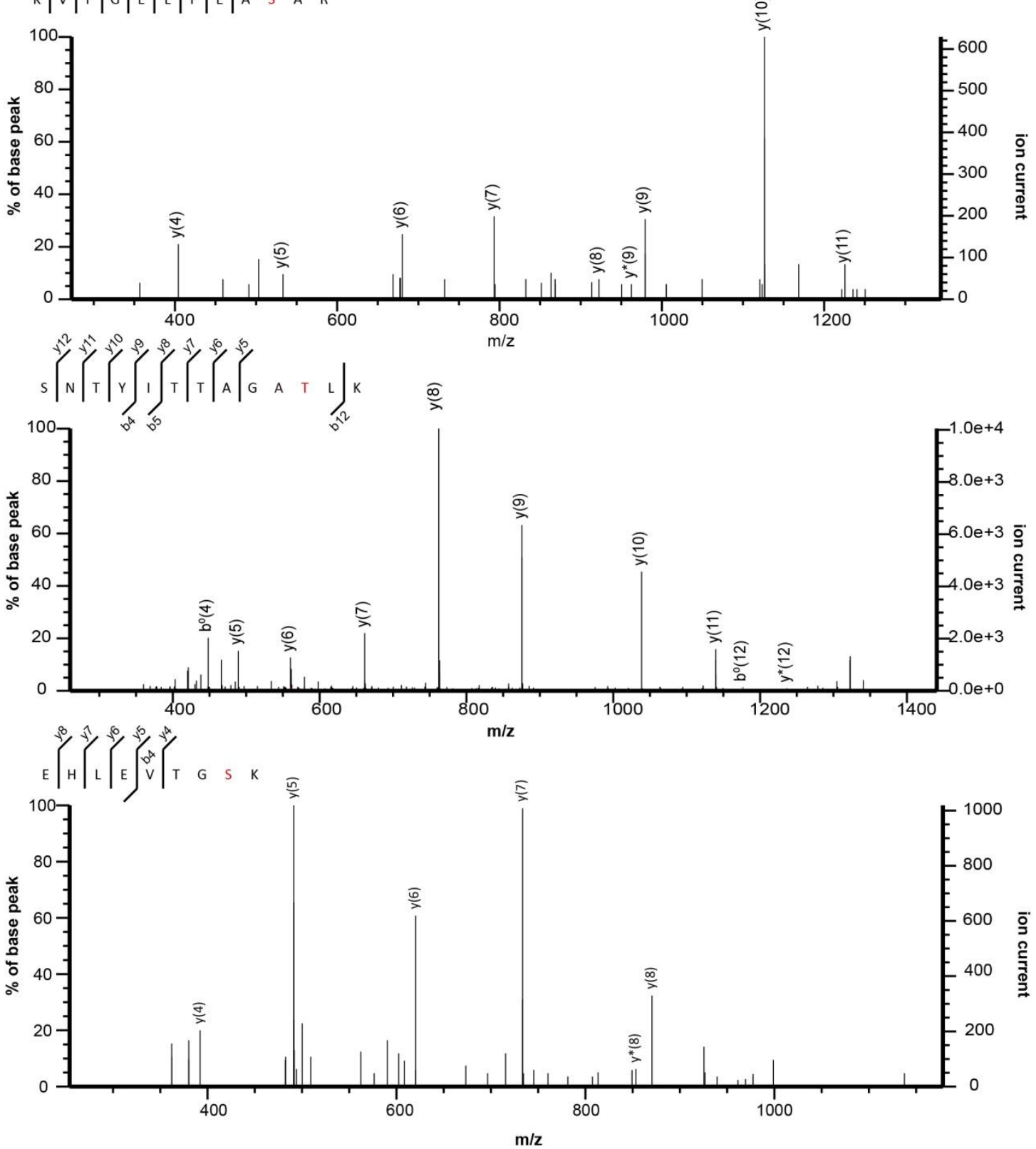

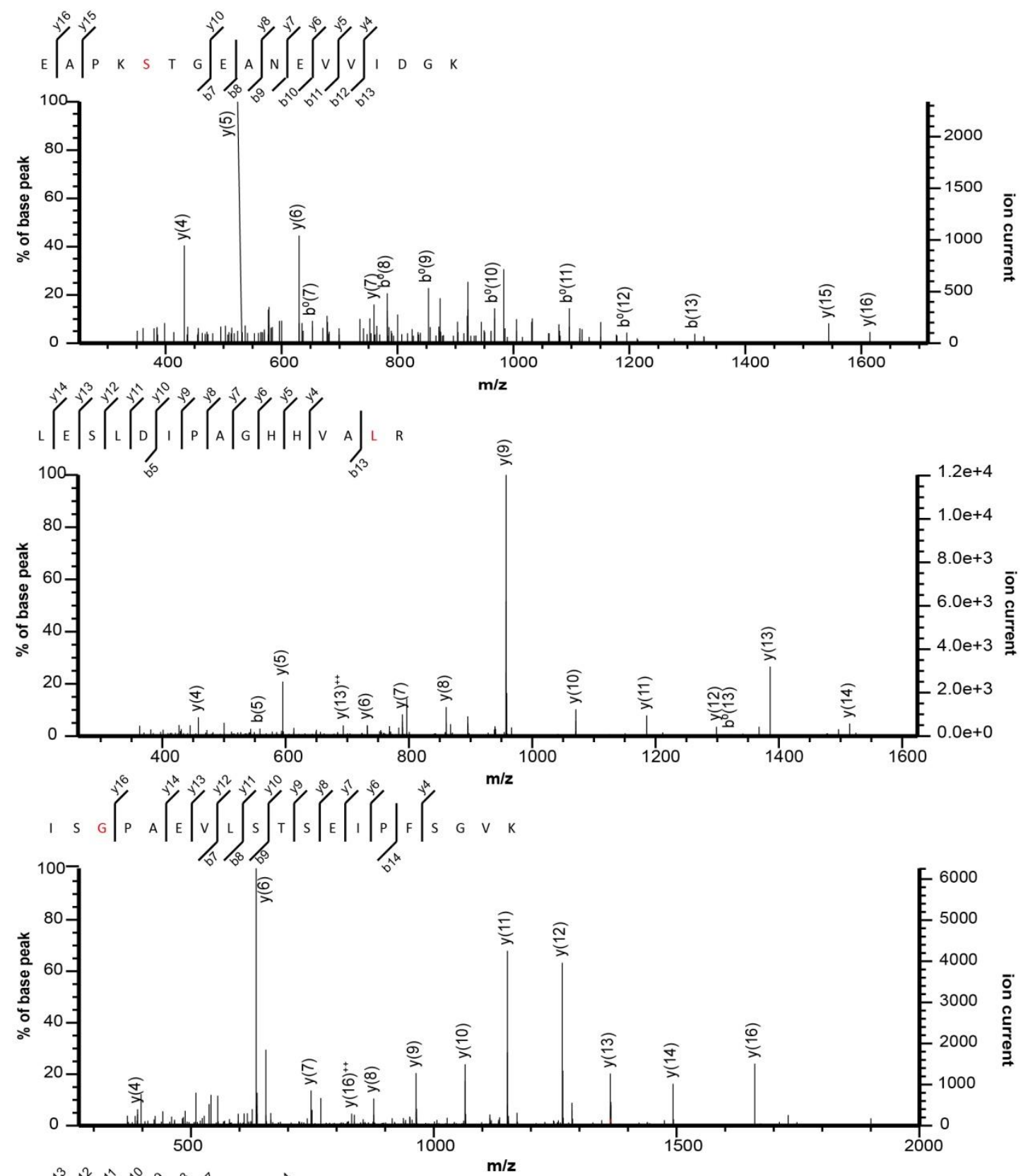

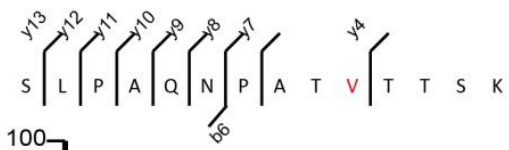

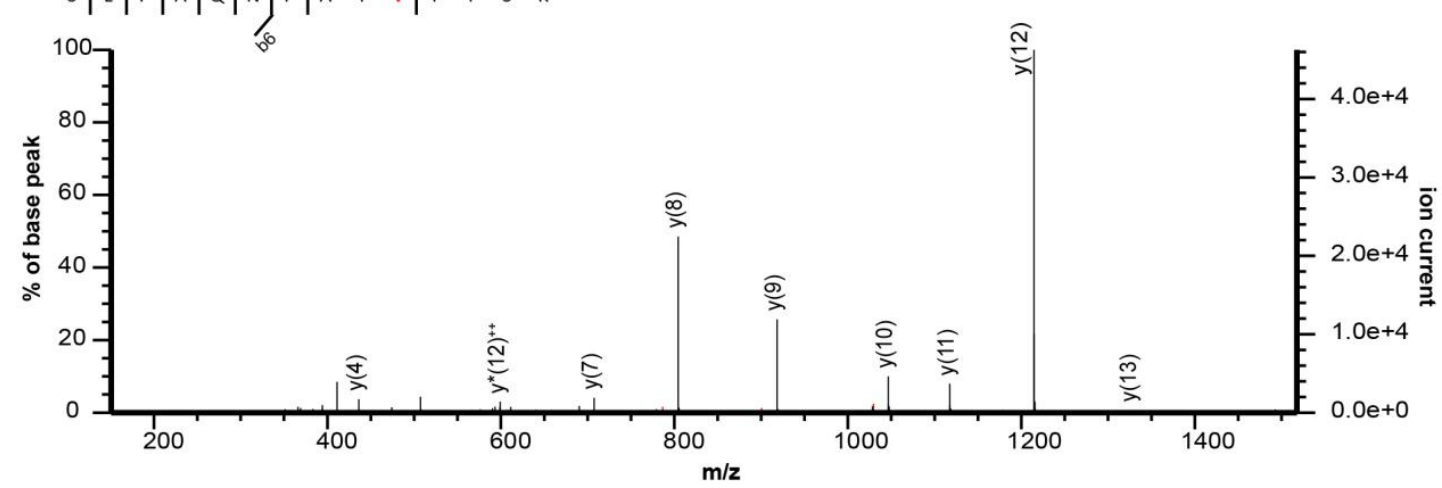


Category 3
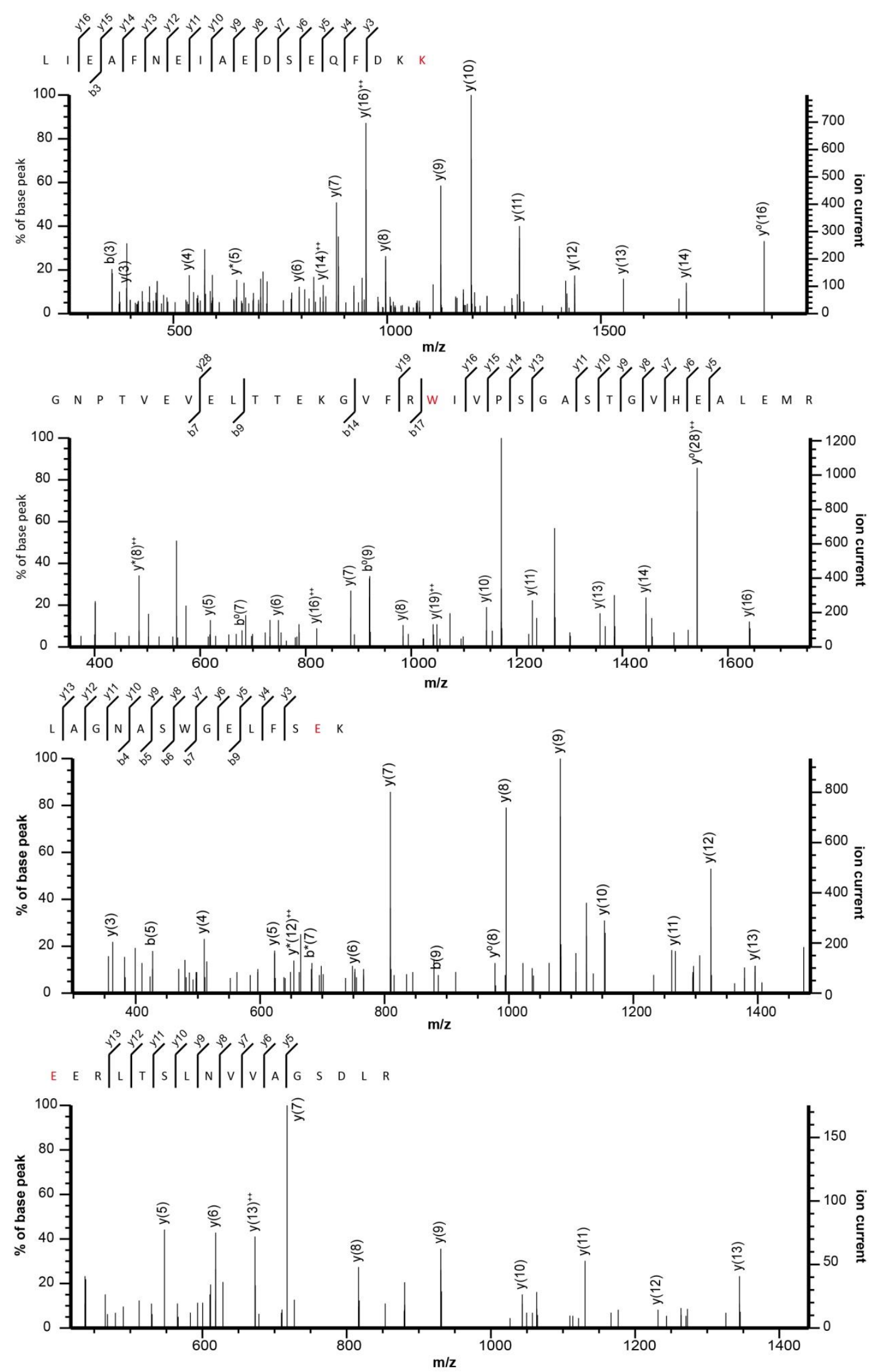

S-8 

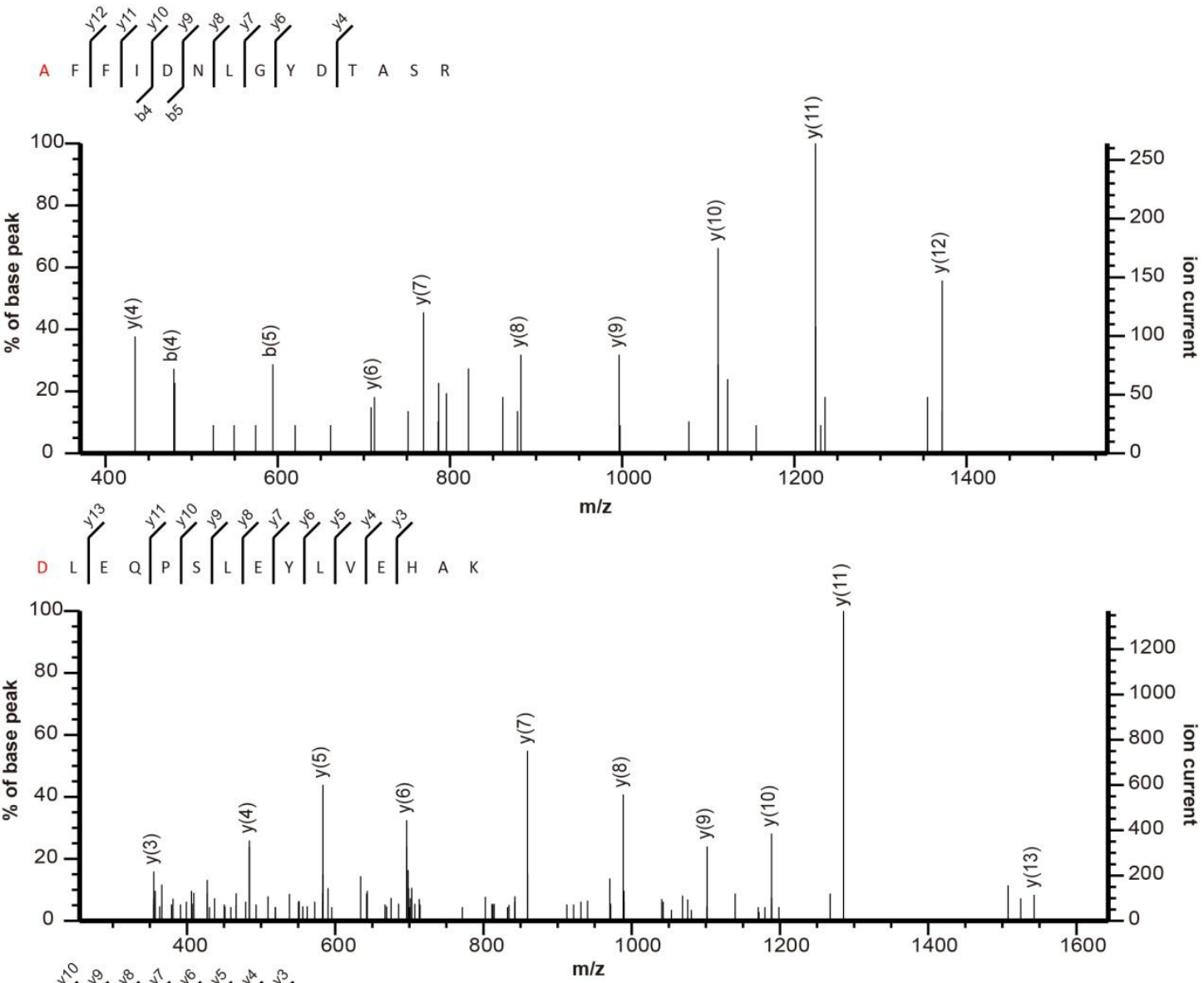

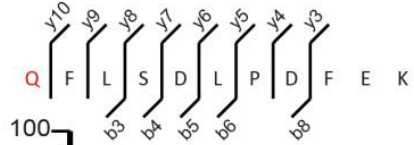
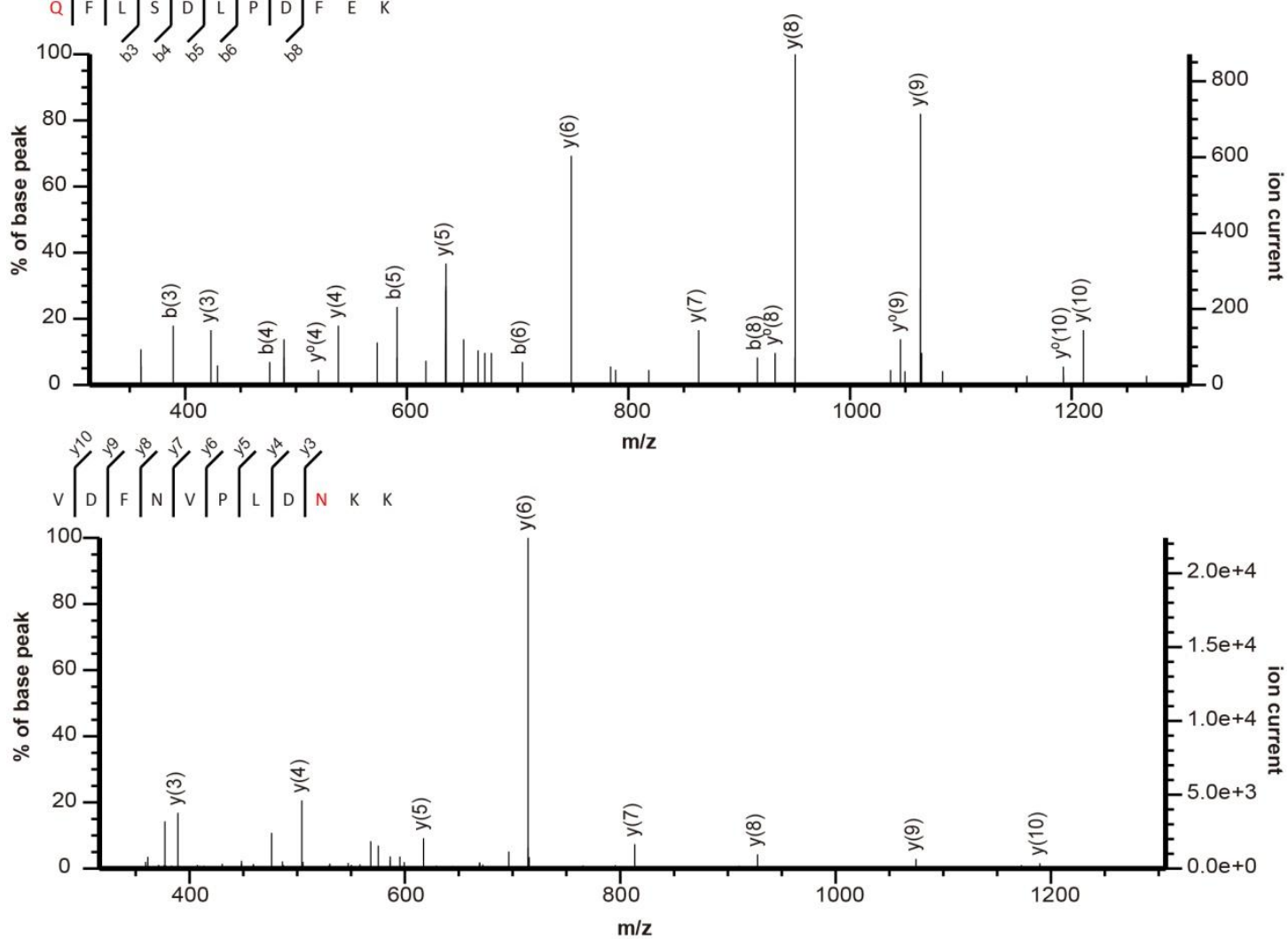

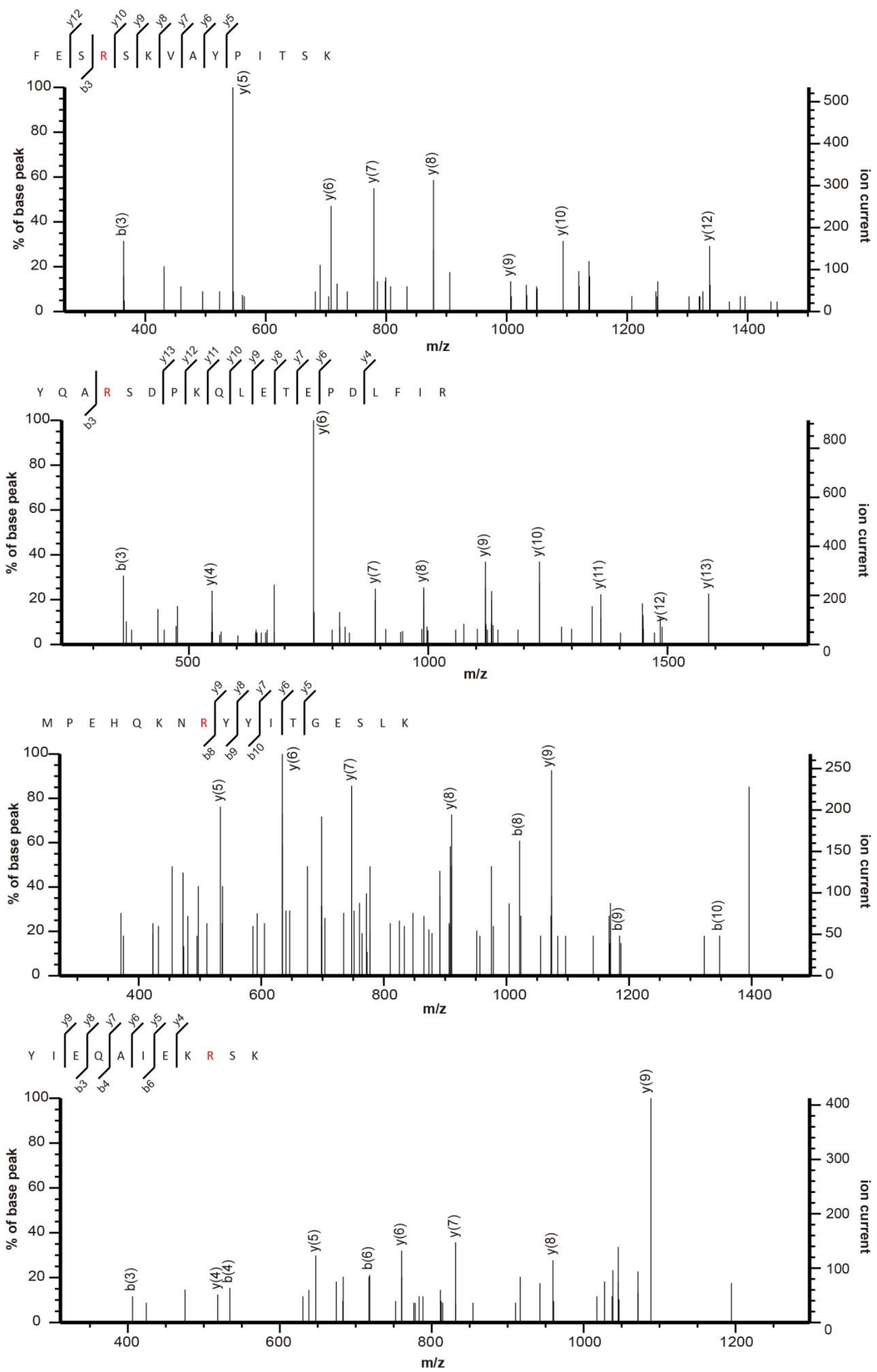

S-10 


\section{Category 4}
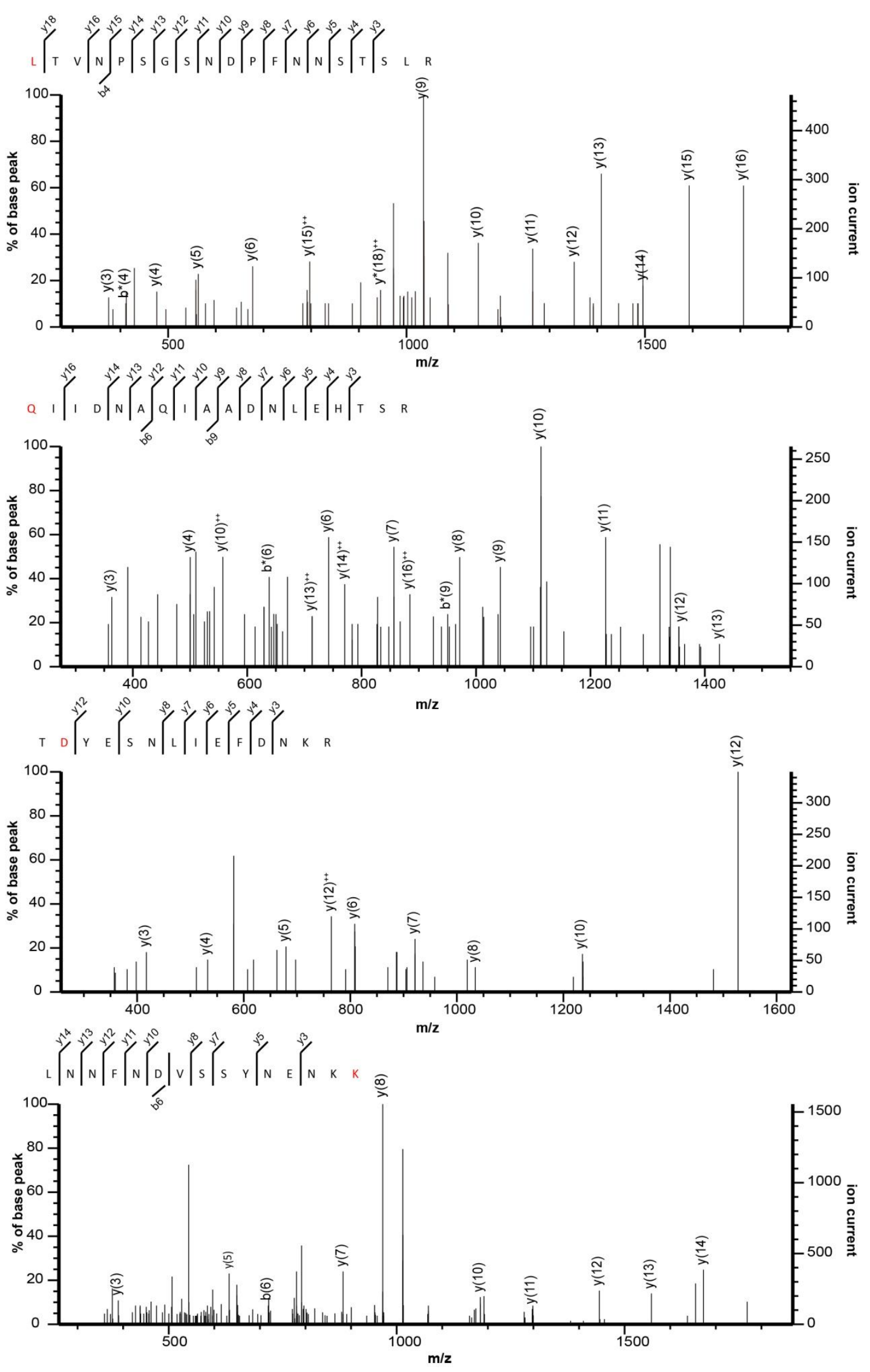

S-11 


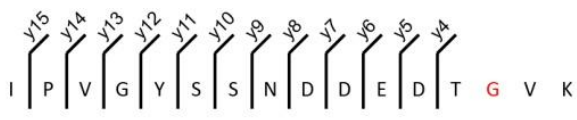

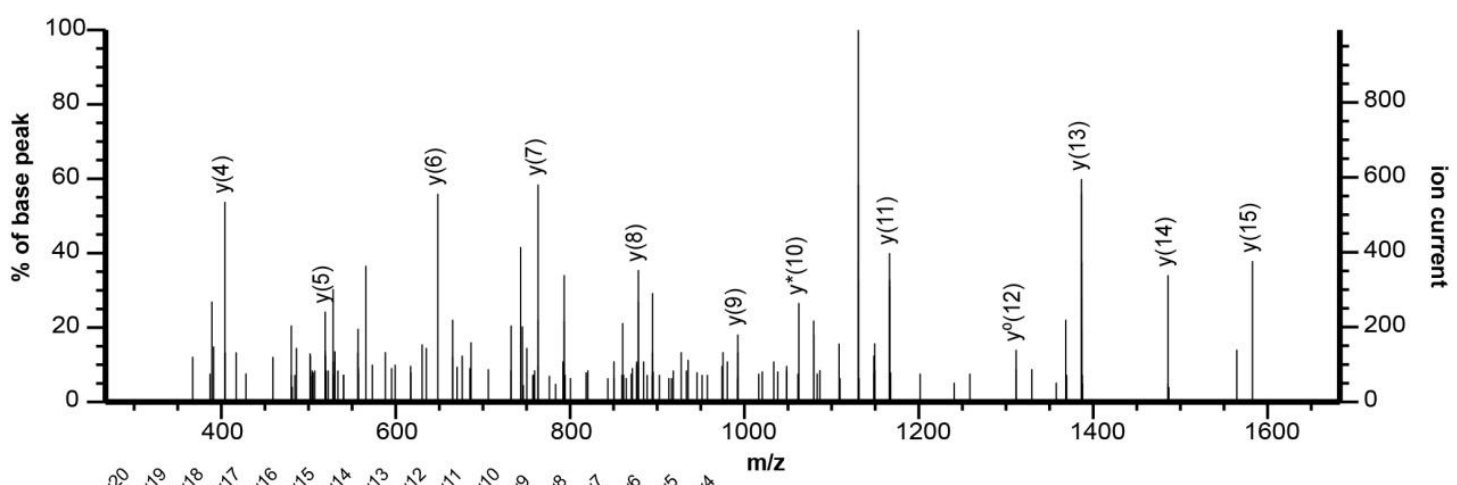

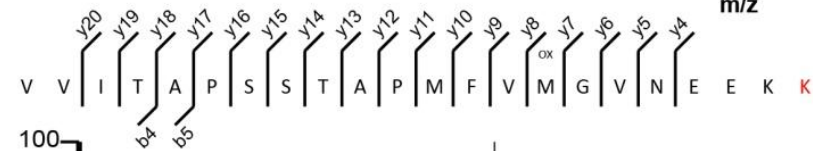
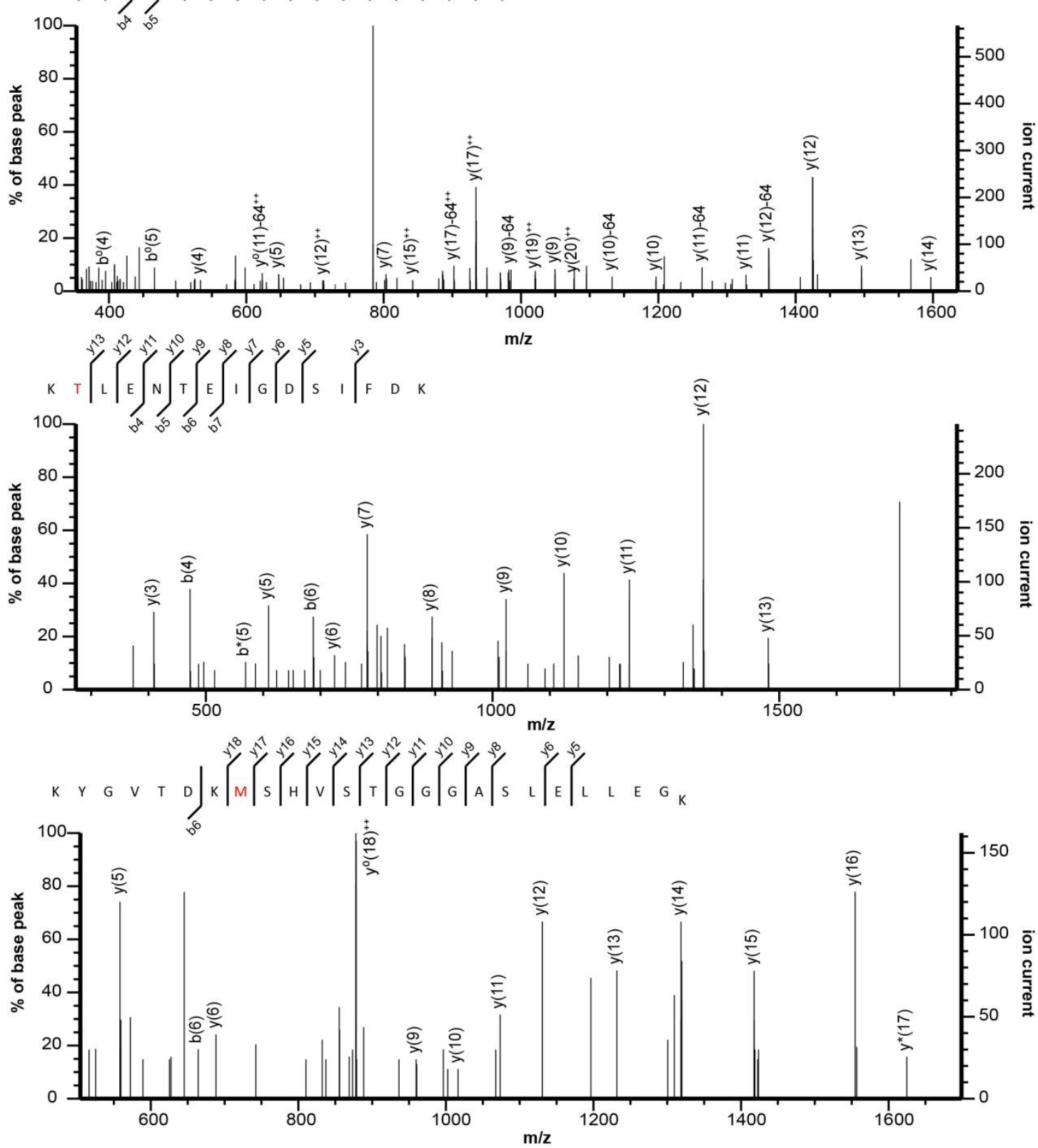

S-12 


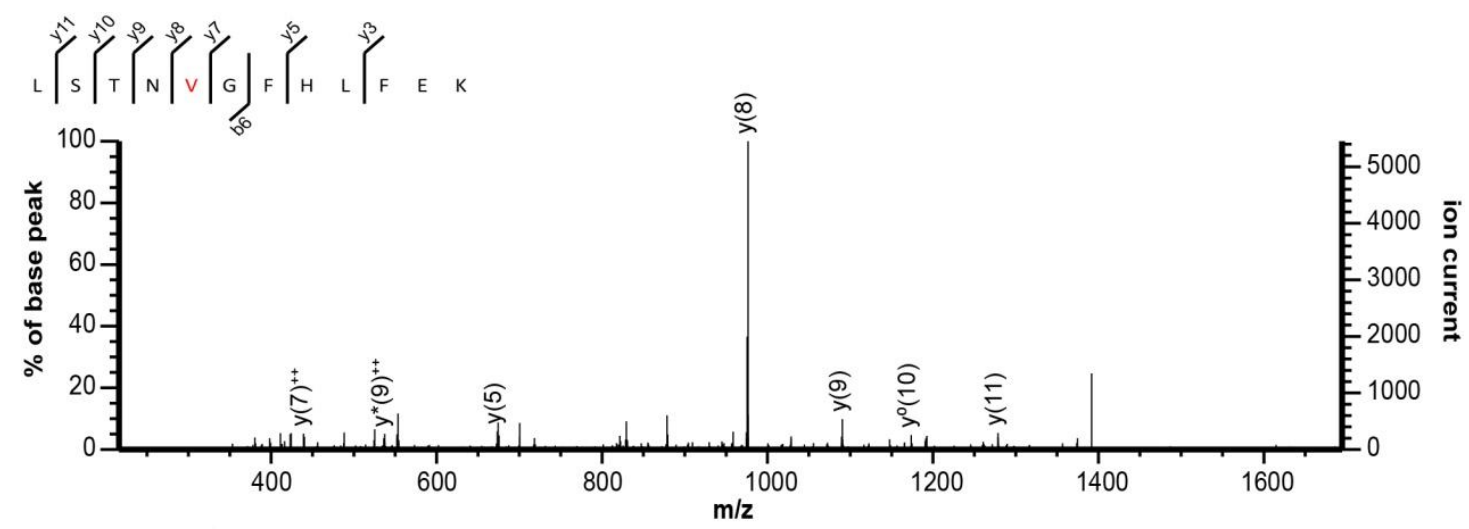

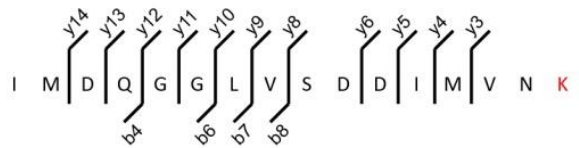

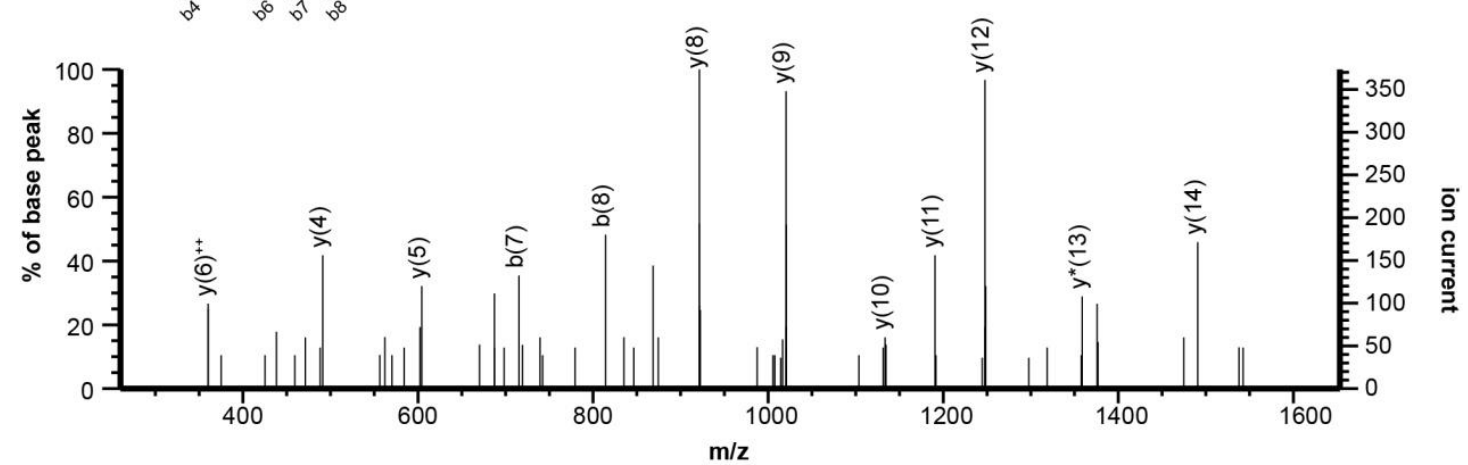

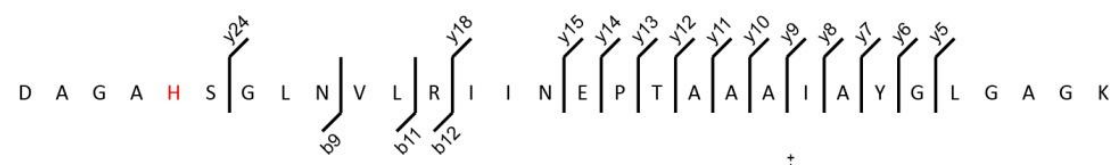

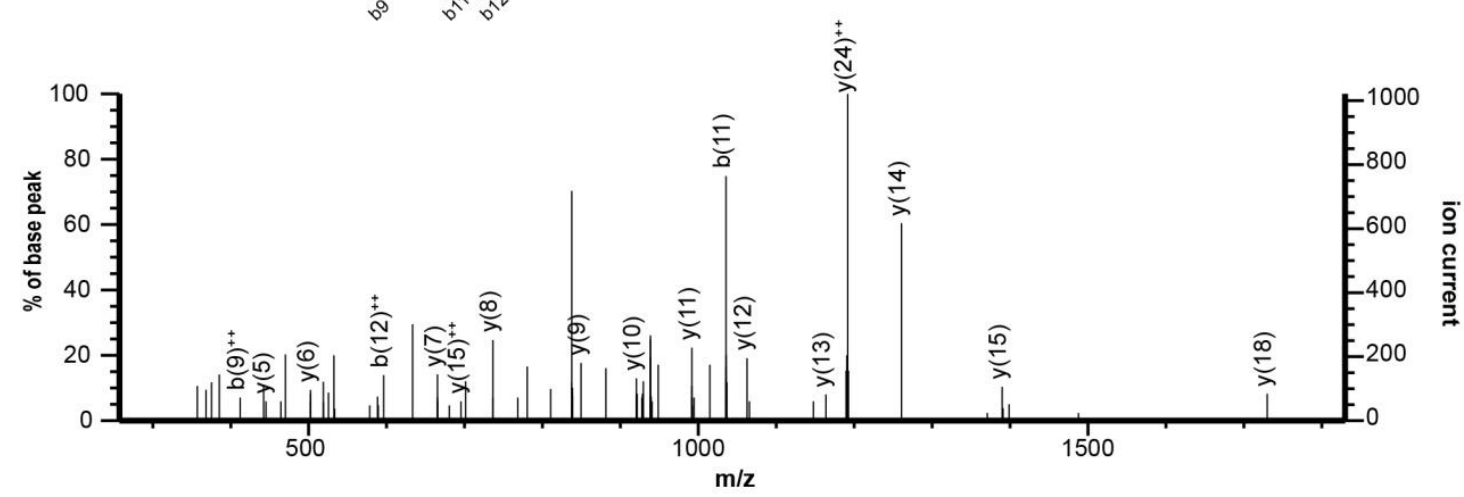

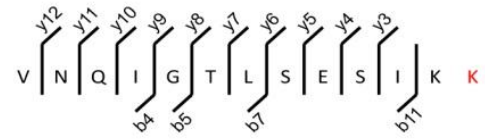

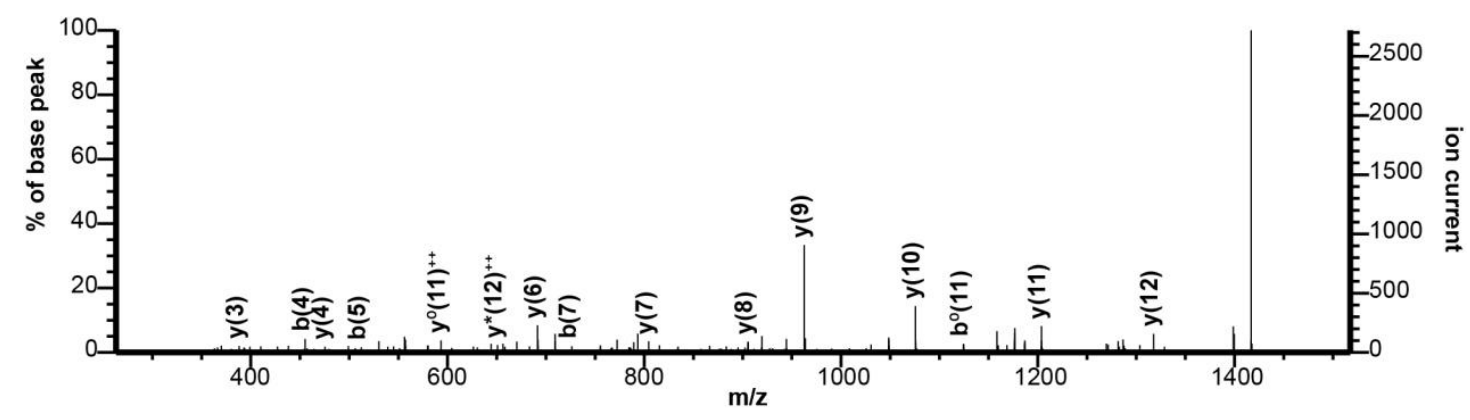

S-13 

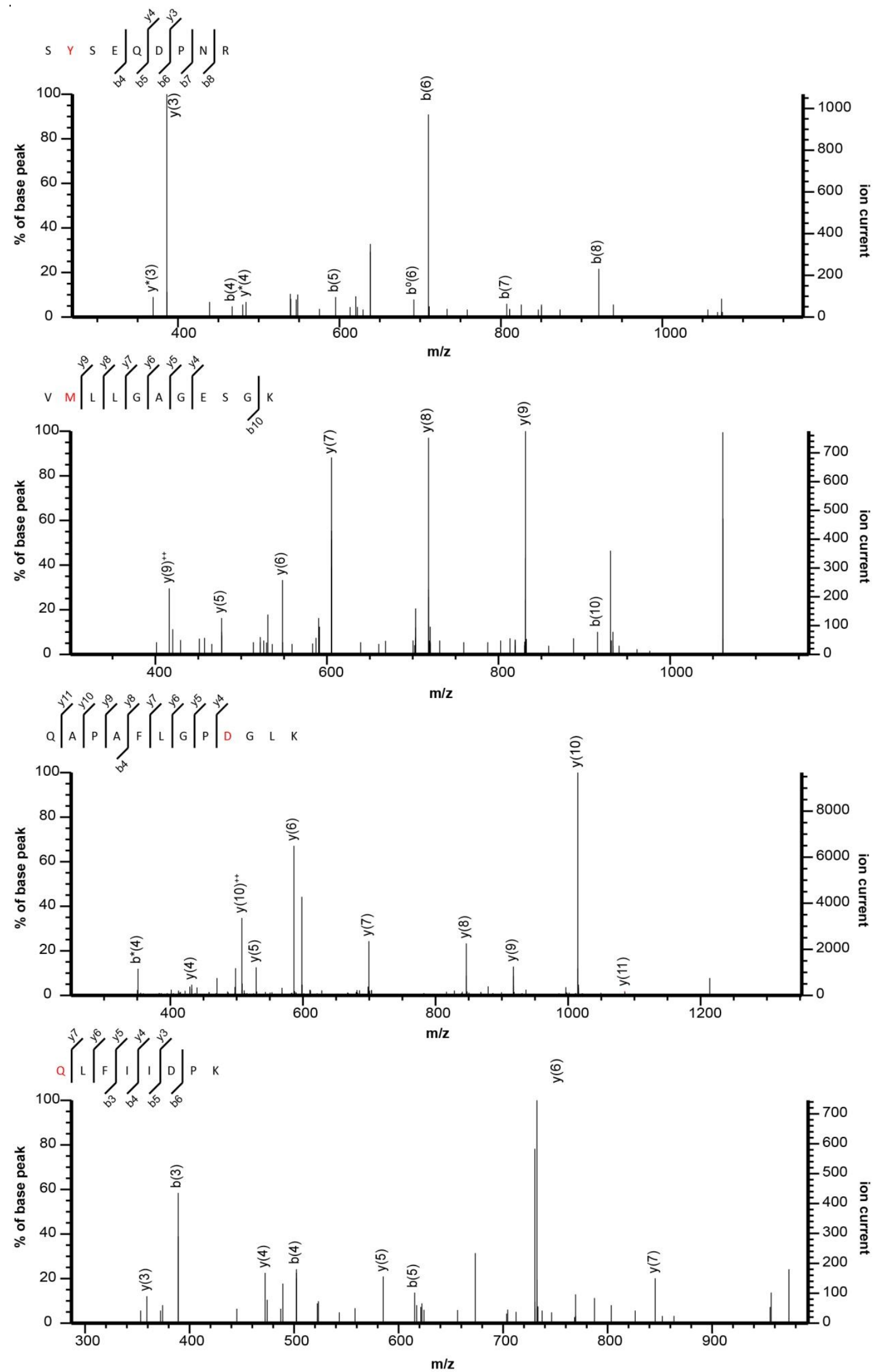

S-14 

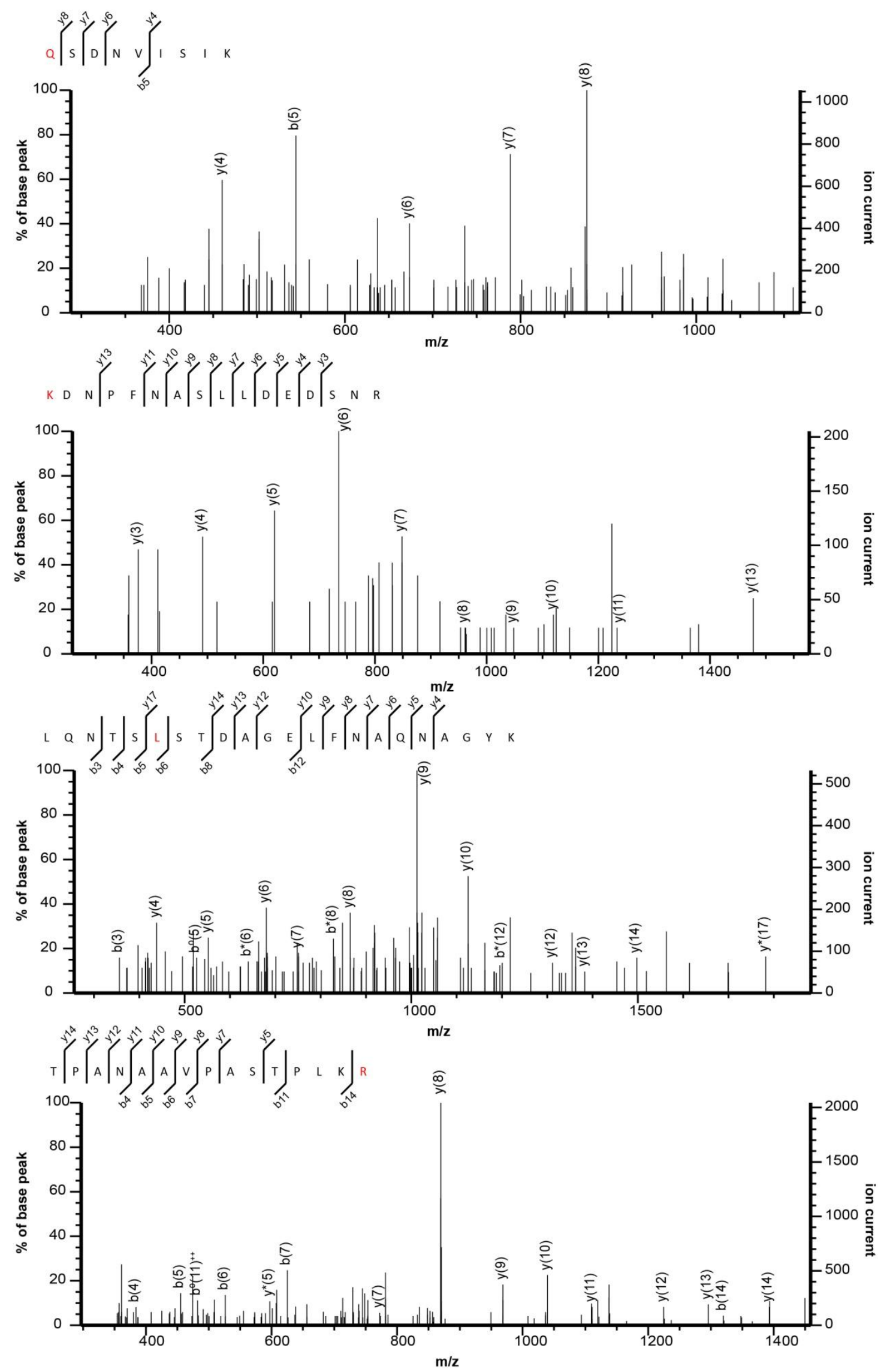

S-15 

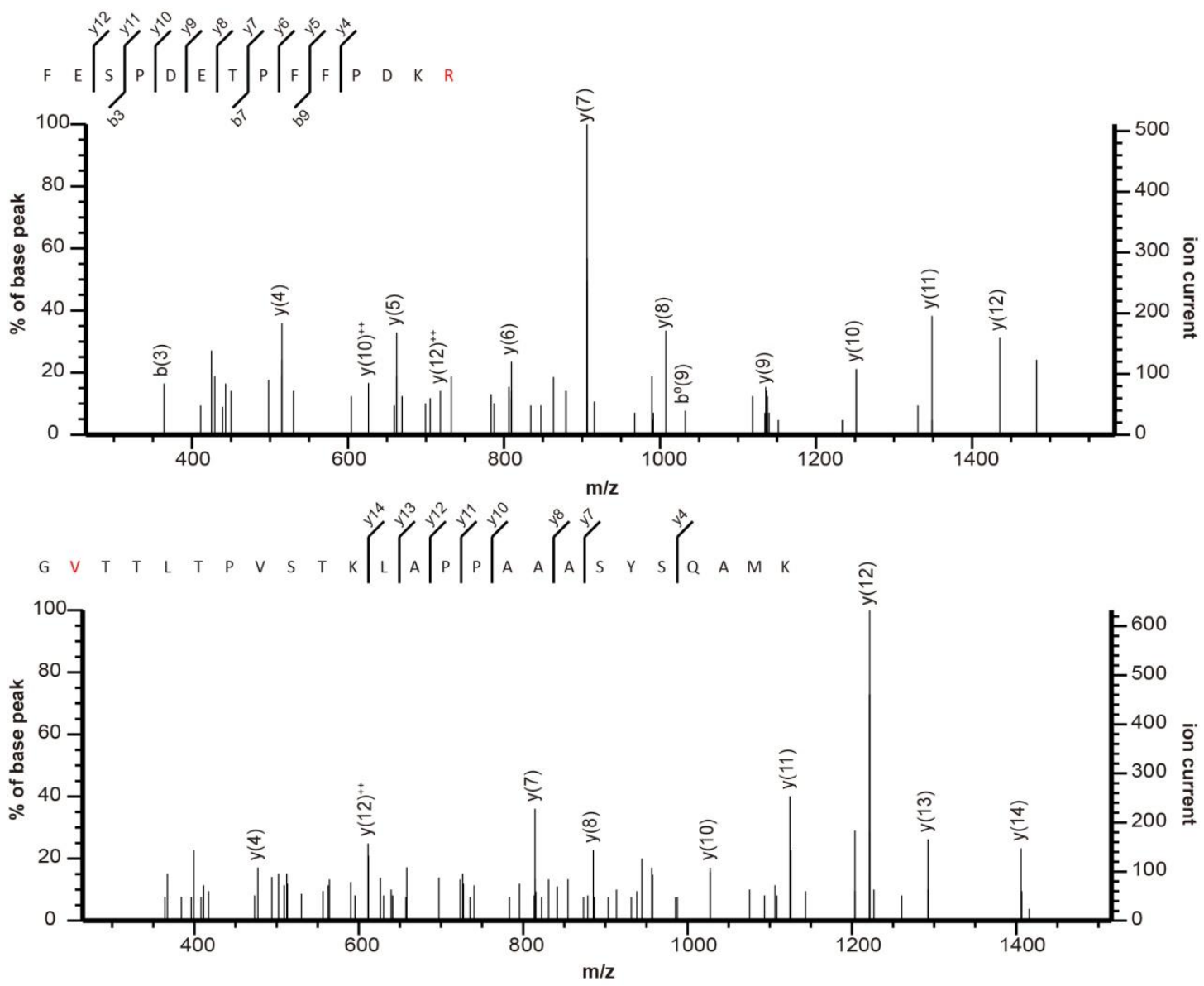

Figure S2. MSMS spectra of 50 non-mapping SAP peptides. The SAP residues are marked in red. 
SAP peptide seq: LYGENVVIPMLNPK SAP: T610V

GATAAACT T TACGGAG AGAACGTTGT TAT TCCTATGT TAA ATCCCAAAGATAGT GAGGCATGGTTATCAGGATTTTTGAACGAG! $\mathbf{D} \mathbf{K}$ L

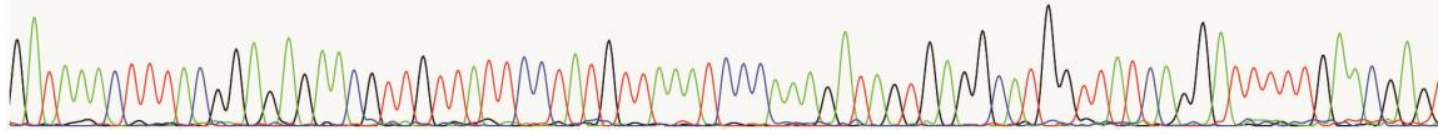

SAP peptide seq: SNFGNNLIDNSSQDK SAP: N584G

ACCAAATCCAAC T T TG GTAATAAC TTAAT T GATAACTCAAG TCAAG ACAAAAT T TCCACGGAGG AAAAATC TTTGTTTTATAAAA $T \mathbf{K}$ S

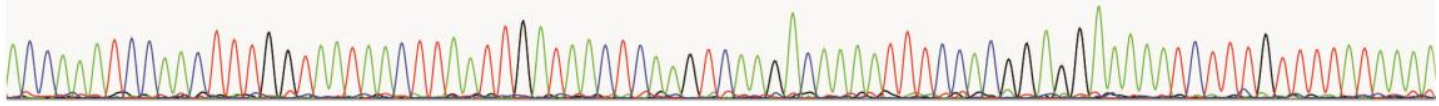
SAP peptide seq: ALEIANALVDVVSK SAP: S157P

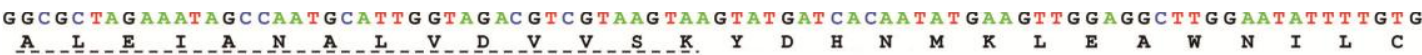

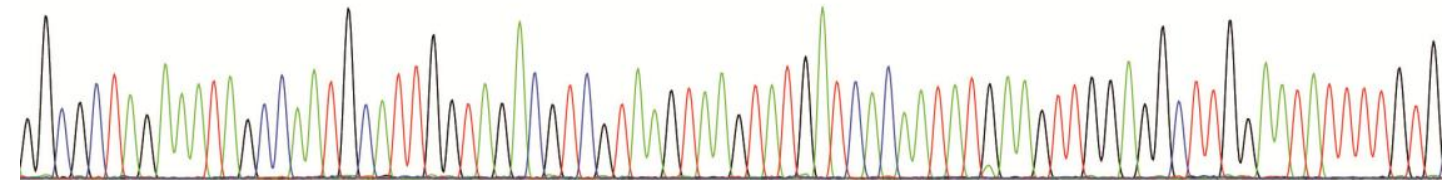

SAP peptide seq: MSDALLLLR SAP: A31L

G G GAAAAT GT C C GACGCAC T GC T GC T GCT GC G TCA GCA GCAGCAG ACCA GC GT G GAT GT G GAGC T GC T GCACACGATGCTAGC GC

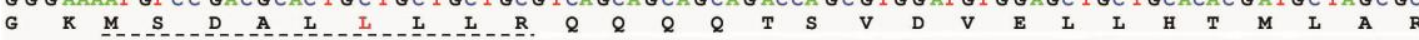

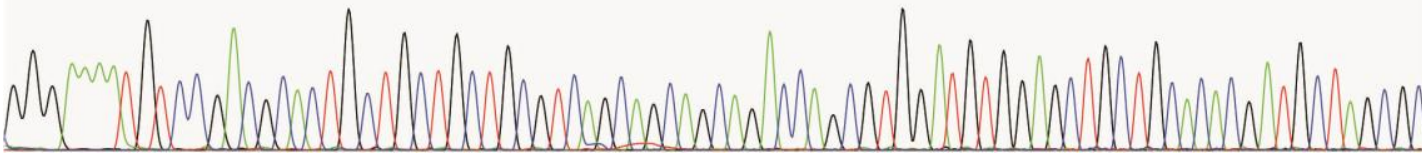

SAP peptide seq: NETPAAQEQPTSDNEPK SAP: M339T, P340S

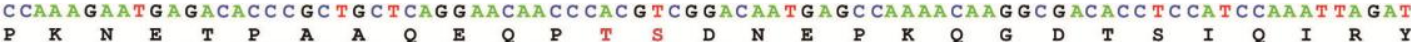

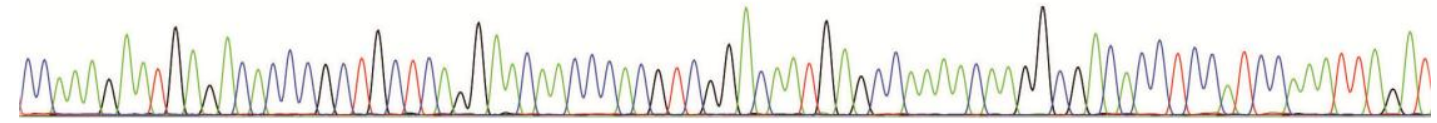

SAP peptide seq: MPEDTTDVEQVASHNDNEGNPSEAK SAP: P313H, S318G

AATAAGATGCCCGAGGACACAACAGATGTCGAACA GGTTGCATCACATAATGACAATG AG GGTAATCCTTCGGAAGCTAAGGAG

$N \quad K \quad M \quad P$

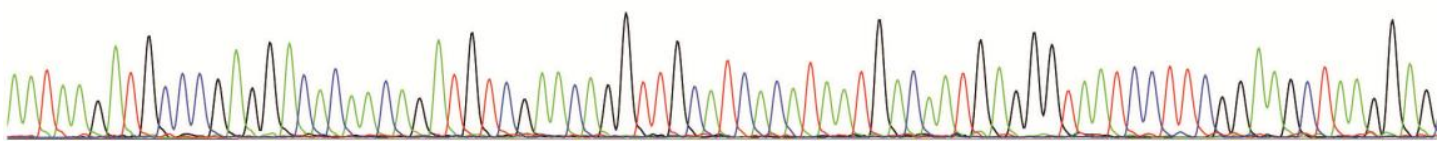


SAP peptide seq: SEESKENSENLTEEQSEIK SAP: N57S, D59N

GCCAAAAGT GAAGAAT C CAAAGAAAACA GT GAAAATT T GACTGAAGAGCAATCAGAAATCAAGAAATTAGAGAGCCA TT TAAGC $\mathbf{A} \mathbf{K} \mathbf{S} \mathrm{E}$

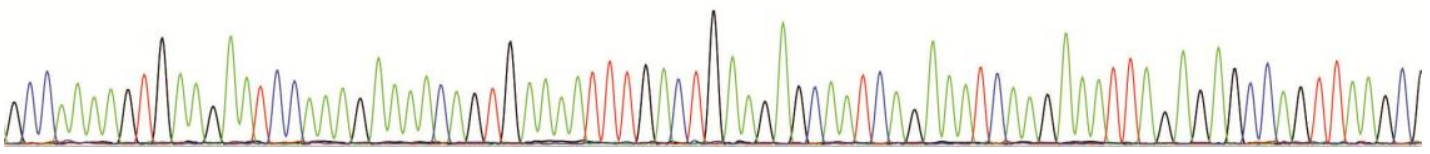

SAP peptide seq: LESLDIPAGHHIAVR SAP: V109I

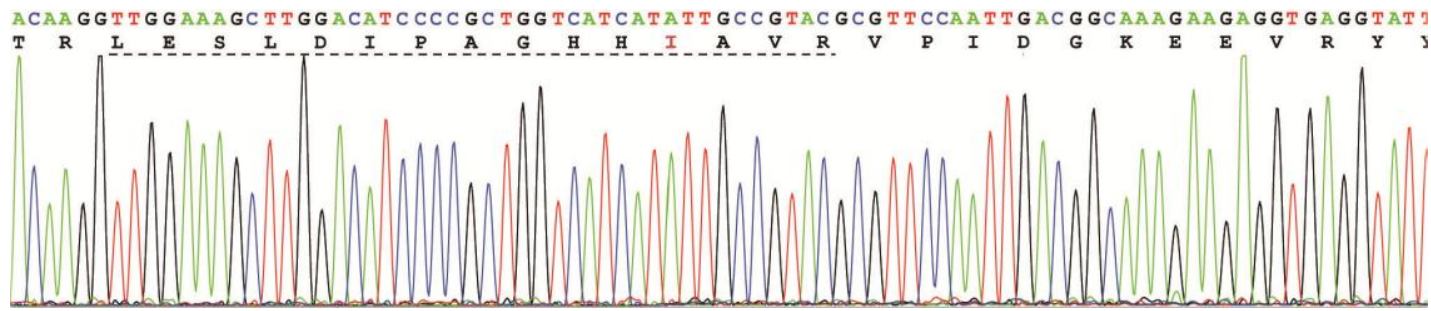

SAP peptide seq: IGSPAEVLSTSEIPFSGVK SAP: S461G

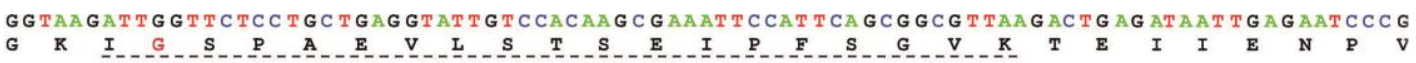

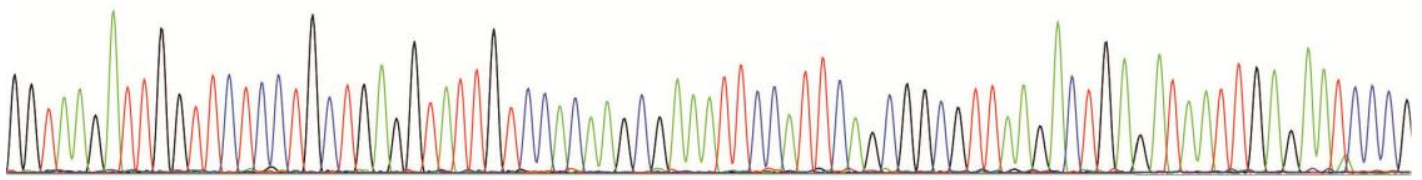

SAP peptide seq: SLPAQNPVTATTSK SAP: A754V

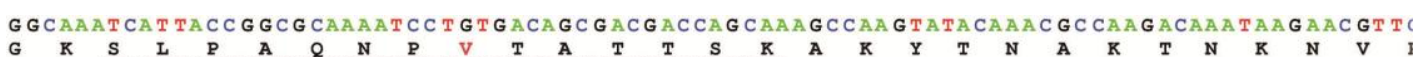

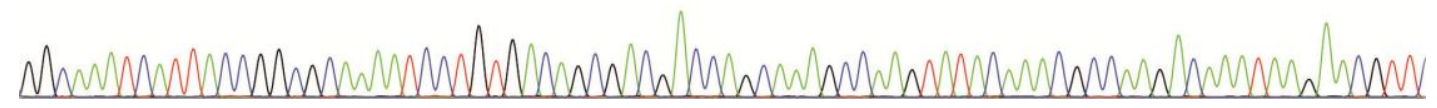

Figure S3. Sanger sequencing confirms the nsSNP sites translating to SAPs in selected peptides. SAP peptide sequences are underlined and the SAP residues are in red. 

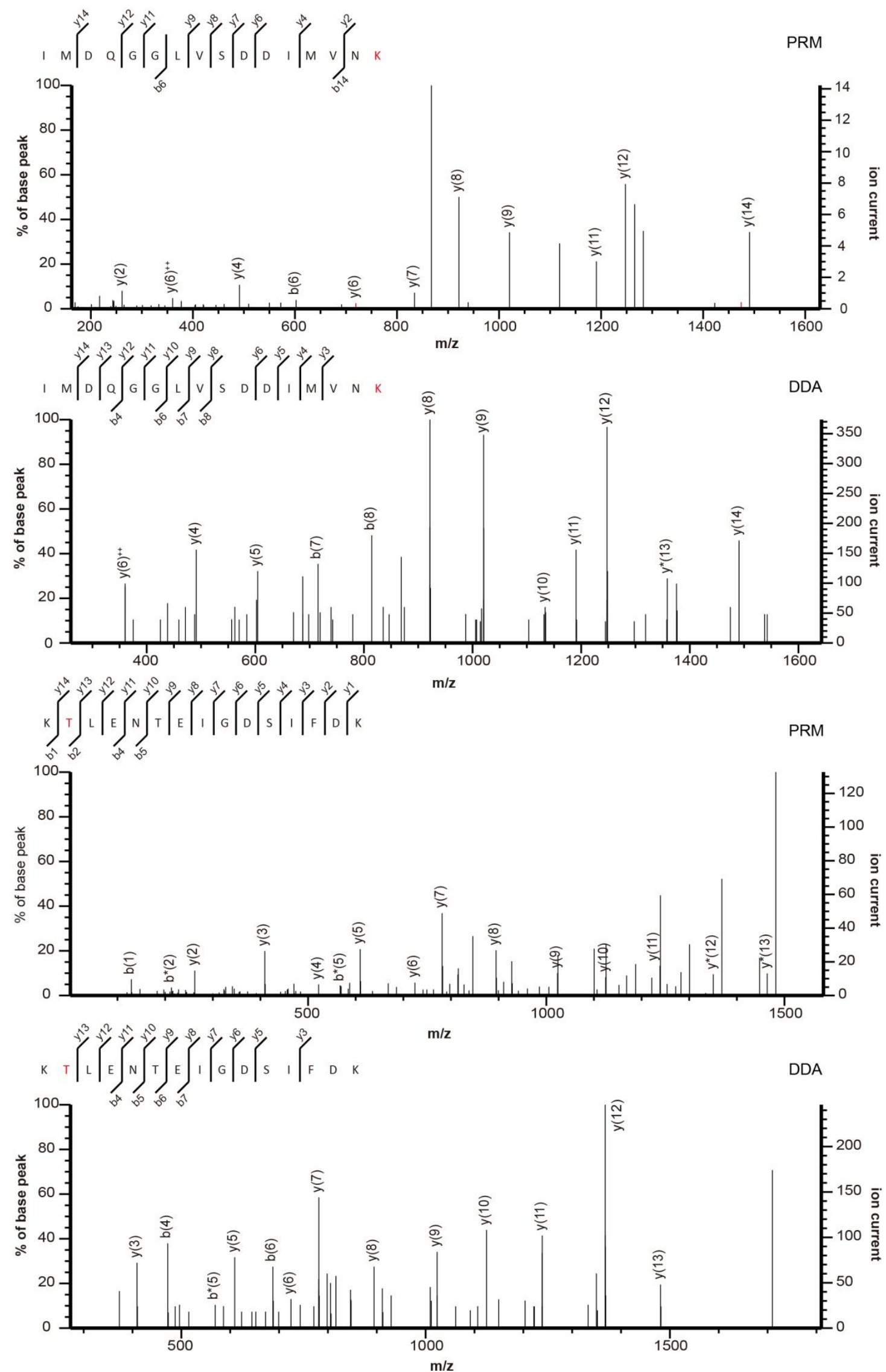

S-19 

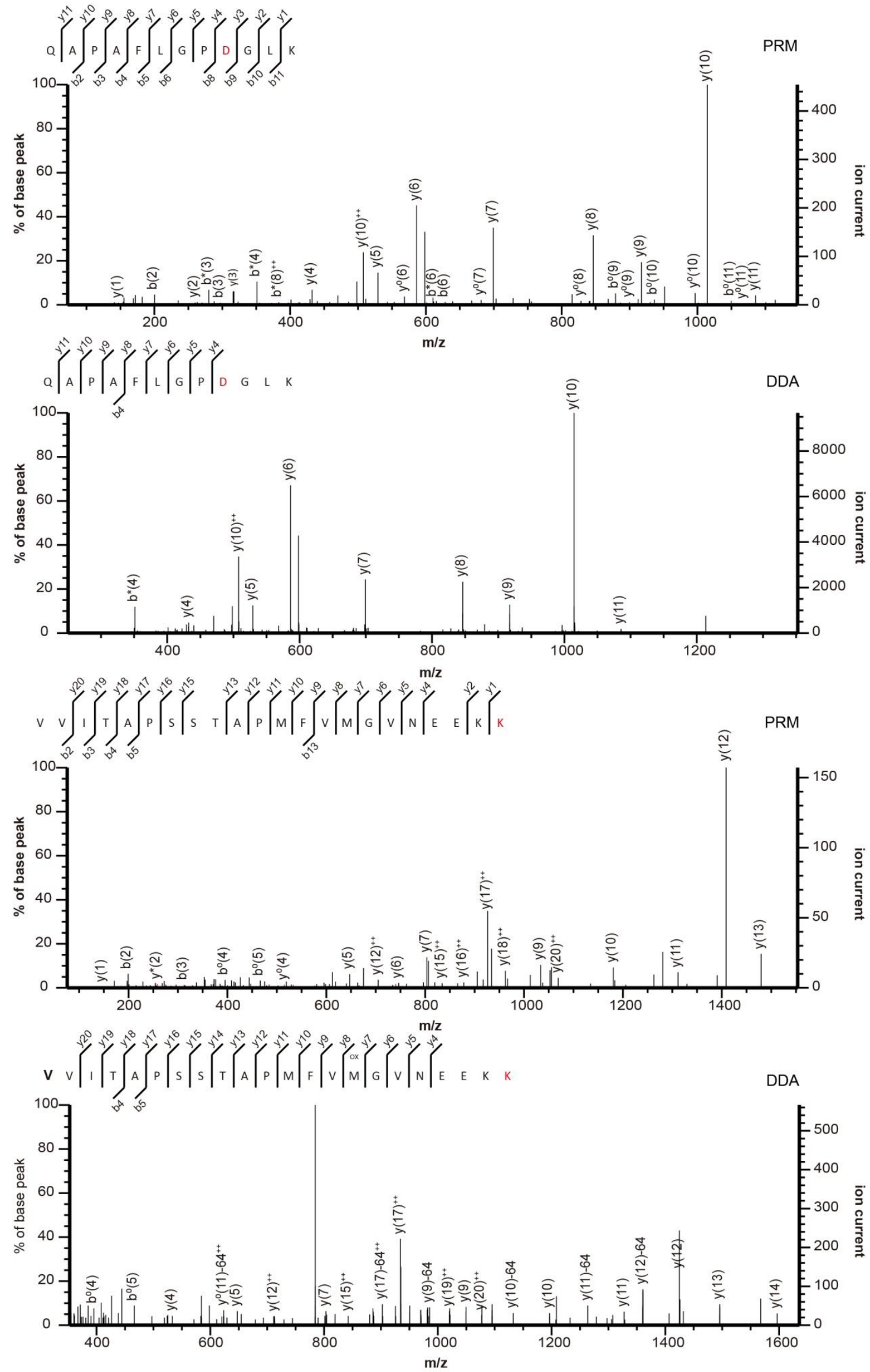

S-20 


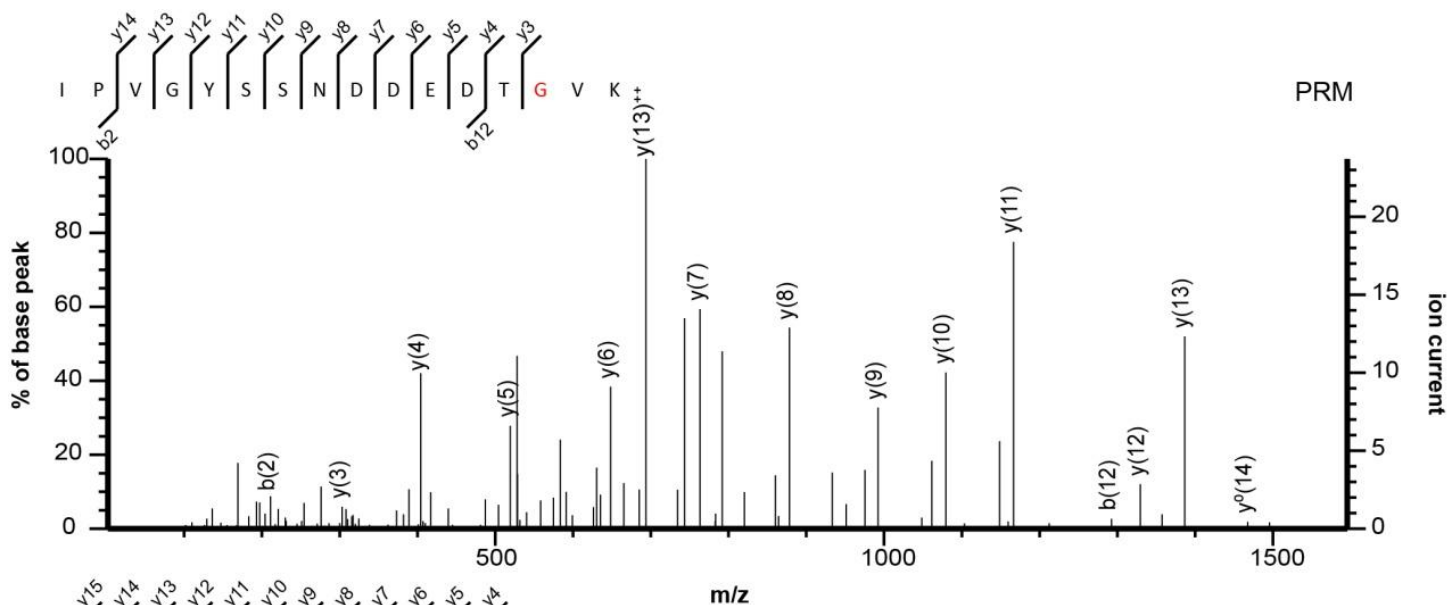

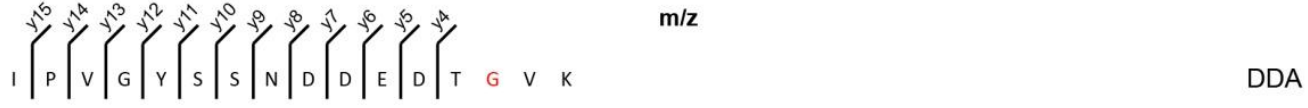

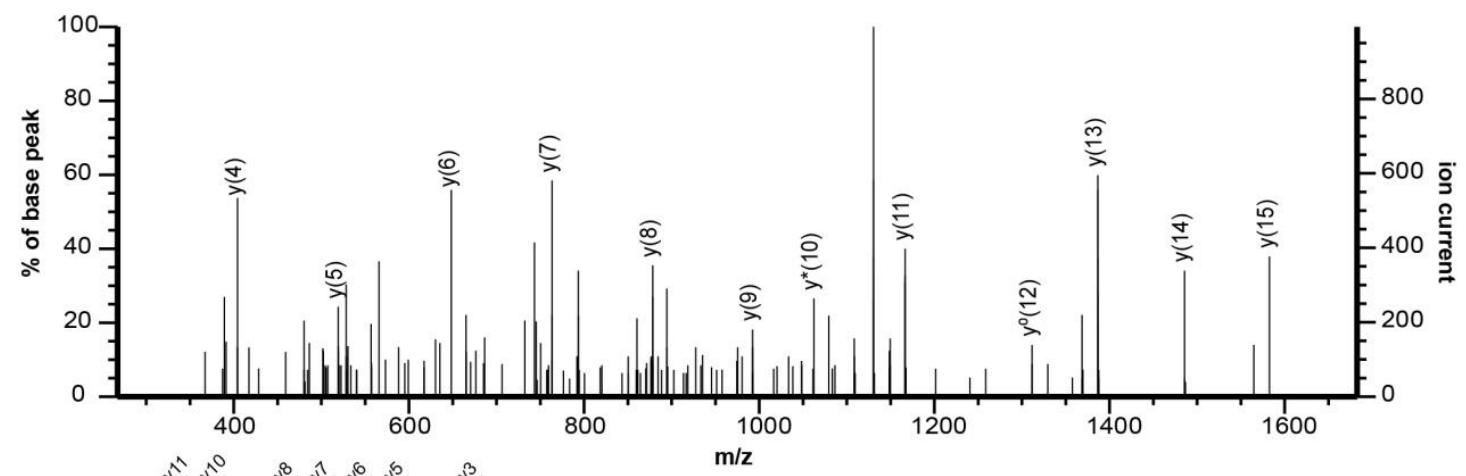

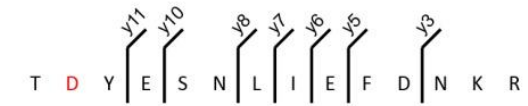
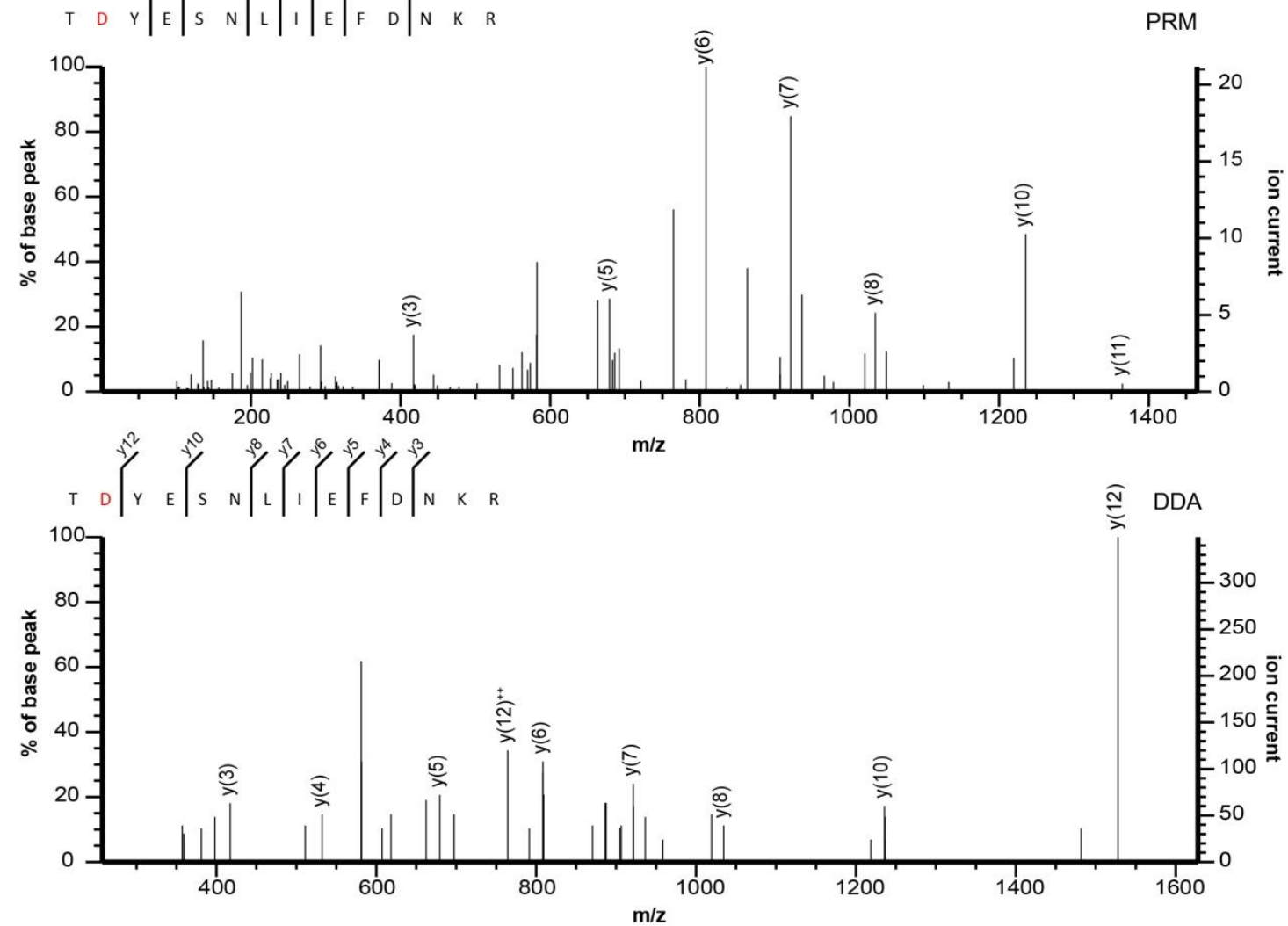

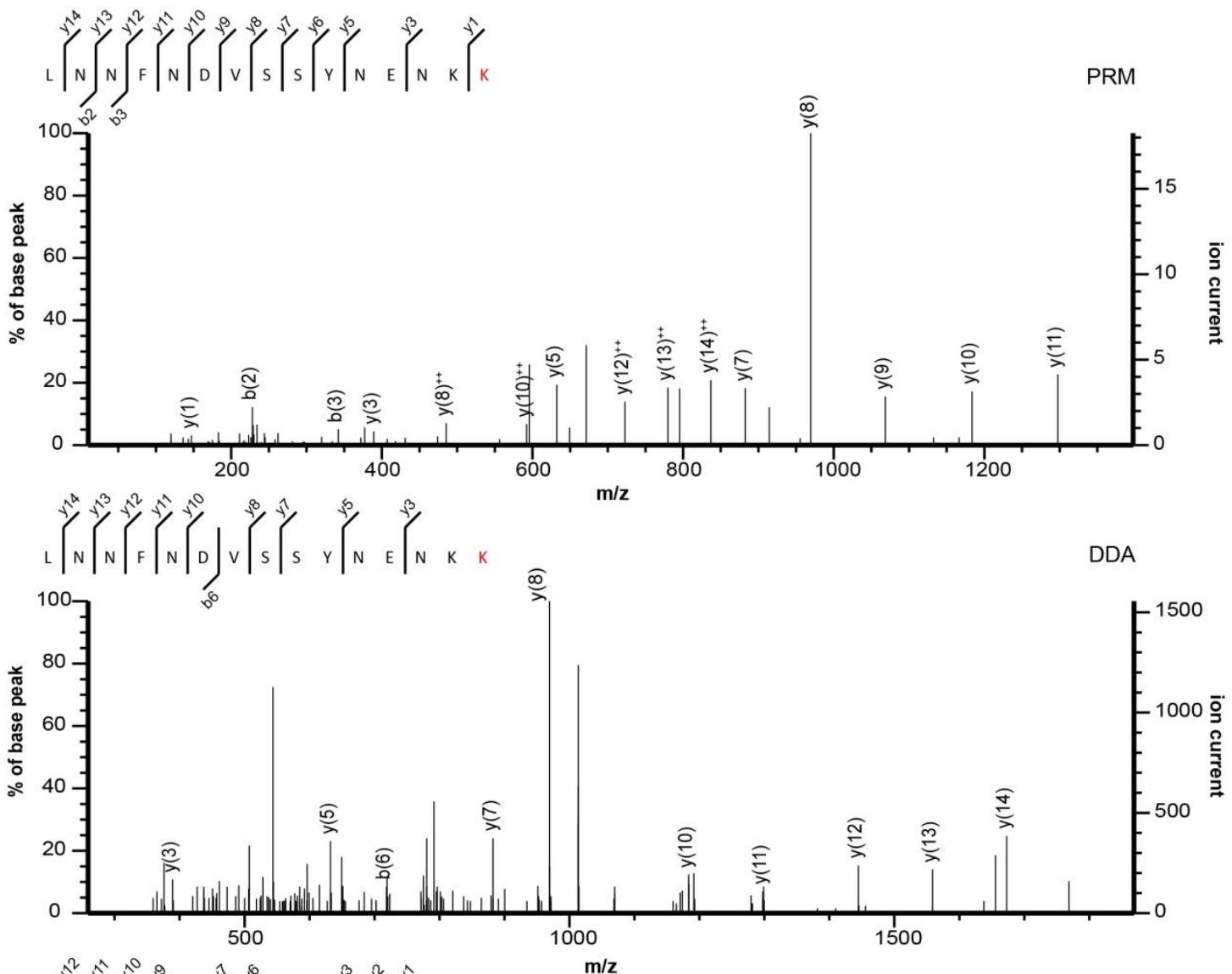

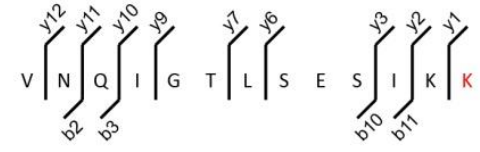

PRM
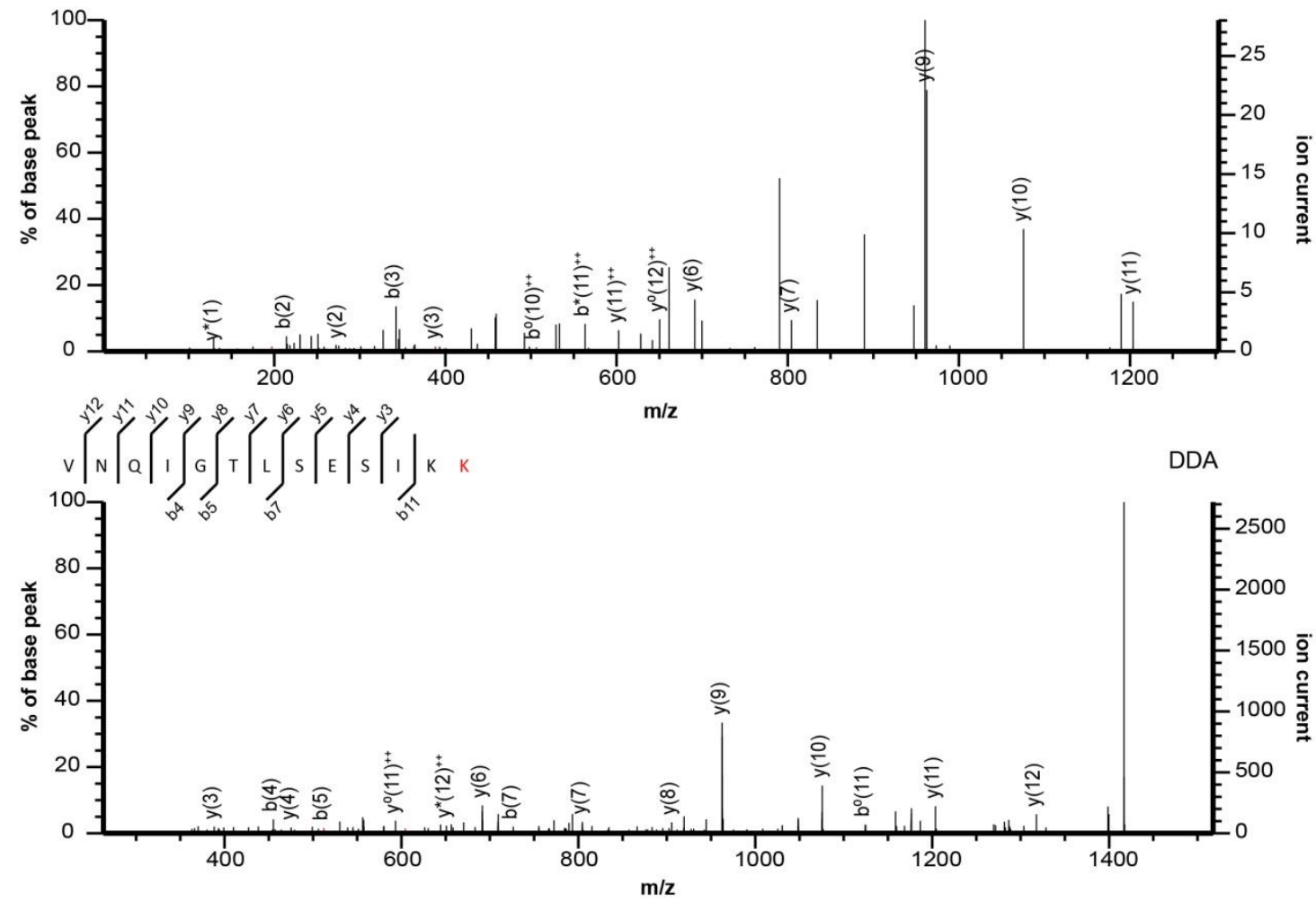

S-22 


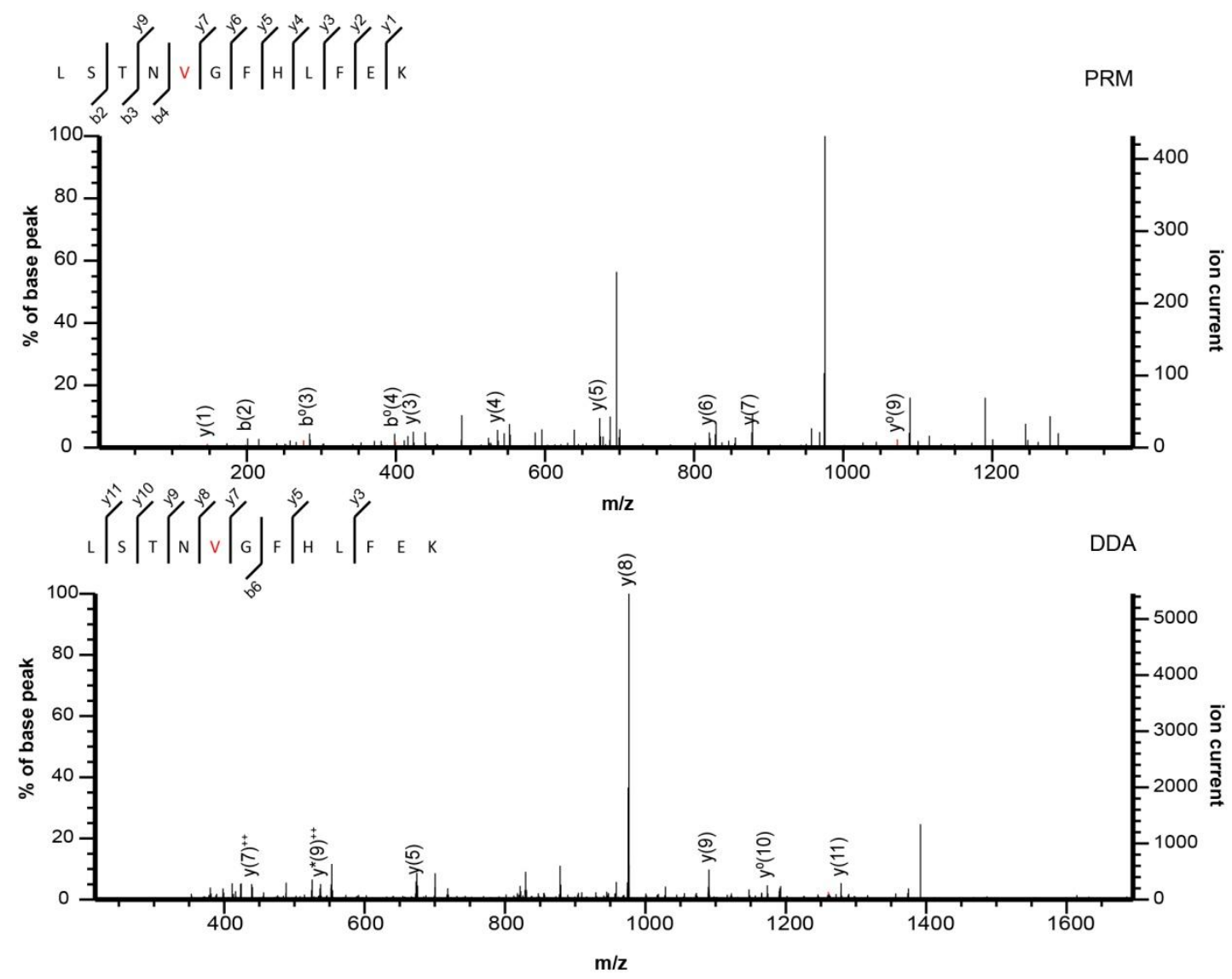

Figure S4. MSMS spectra of nine SAP peptides repetitively identified in PRM analysis (upper) and DDA analysis (lower). SAP peptide sequences and the type of MSMS analysis are shown above the spectra. The SAP residues are marked in red. 

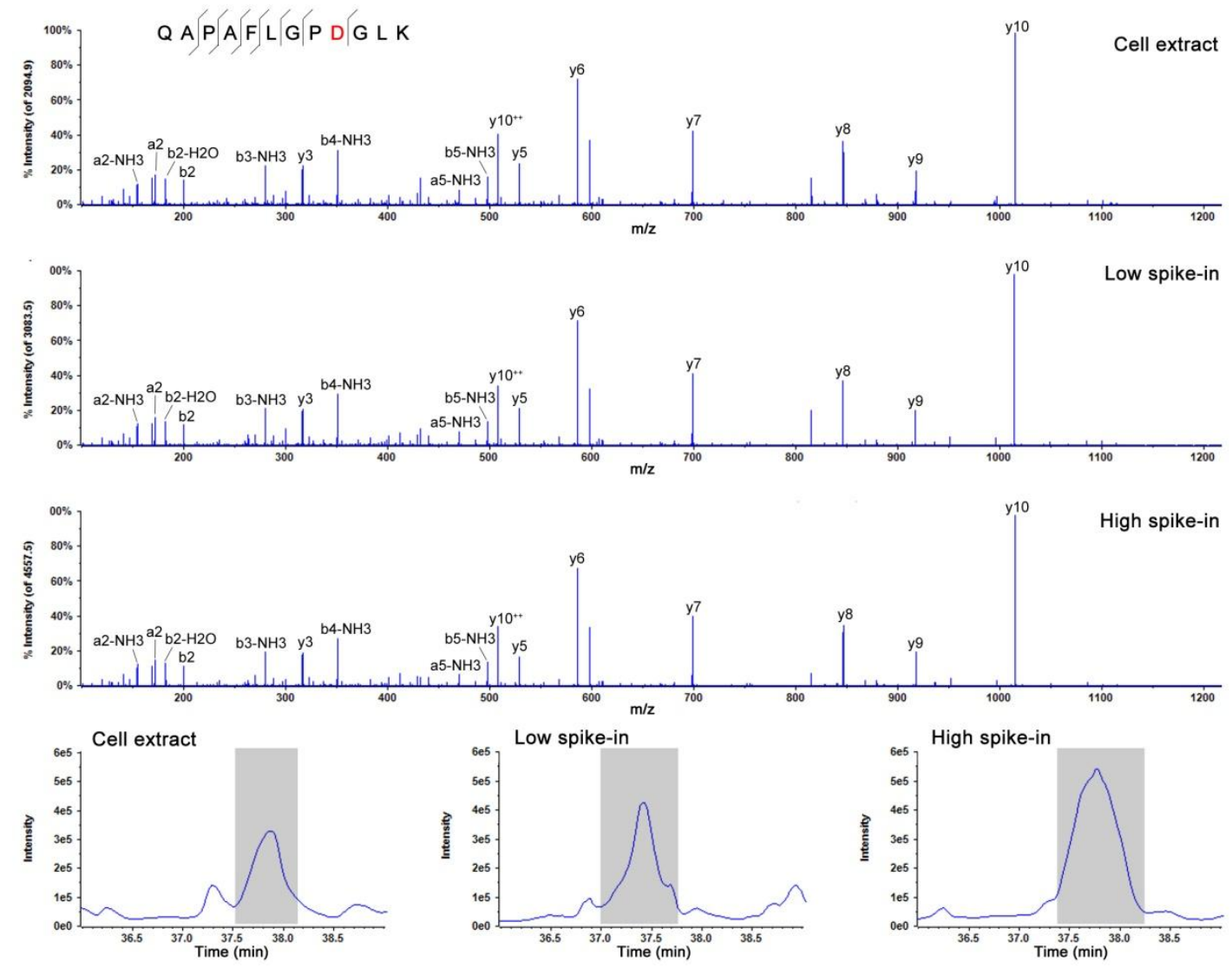

Figure S5. MSMS spectra of the SAP peptide QAPAFLGPDGLK (SAP site in red) detected by PRM analysis in total cell extracts $(A)$, extracts with low spike-in (B) or high spike-in $(C)$ amounts of the synthetic SAP peptide, and corresponding extracted ion chromatograms of the SAP peptide in different samples (D). 

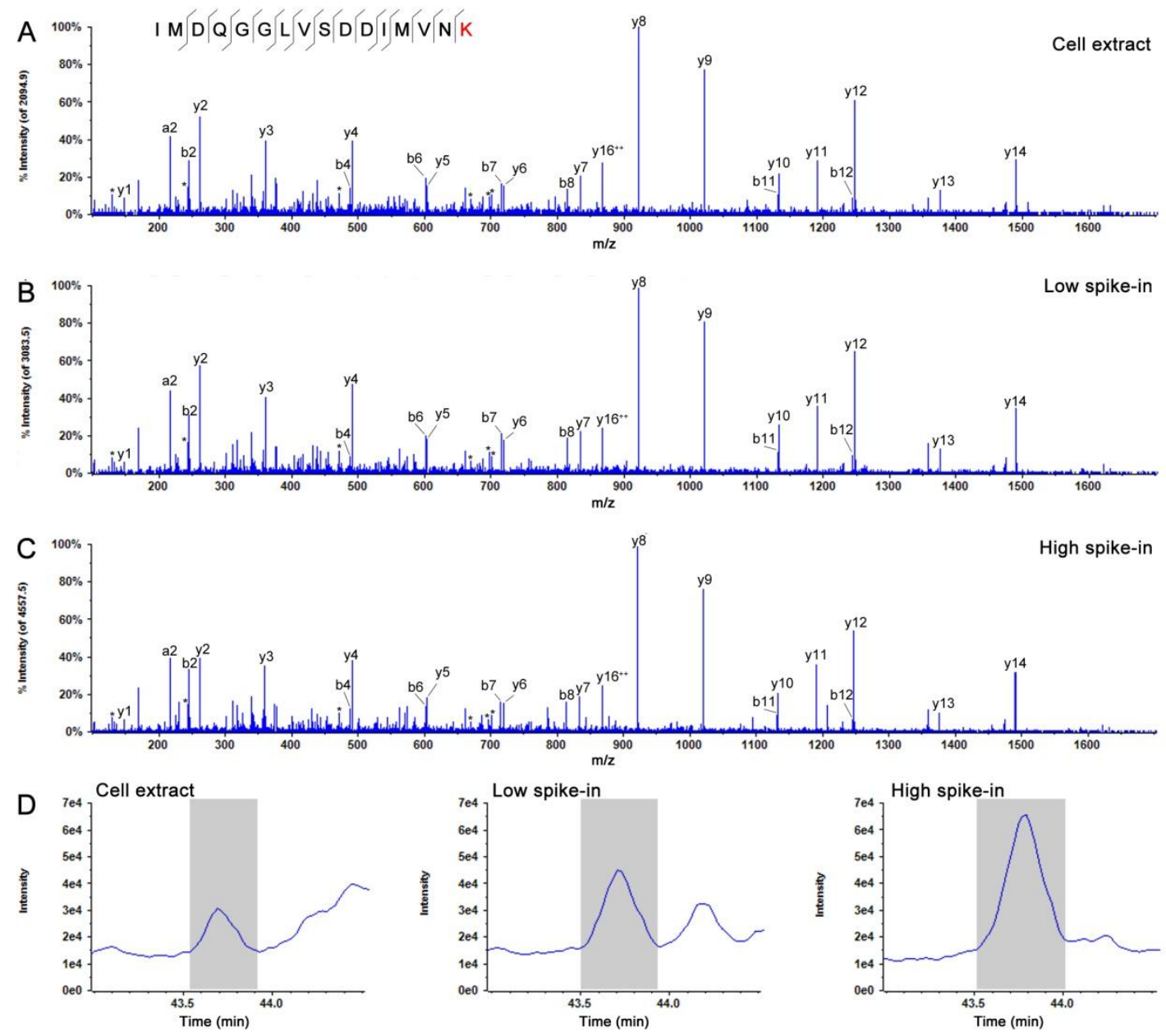

Figure S6. MSMS spectra of the SAP peptide IMDQGGLVSDDIMVNK (SAP site in red) detected by PRM analysis in total cell extracts (A), extracts with low spike-in (B) or high spike-in (C) amounts of the synthetic SAP peptide, and corresponding extracted ion chromatograms of the SAP peptide in different samples (D). 

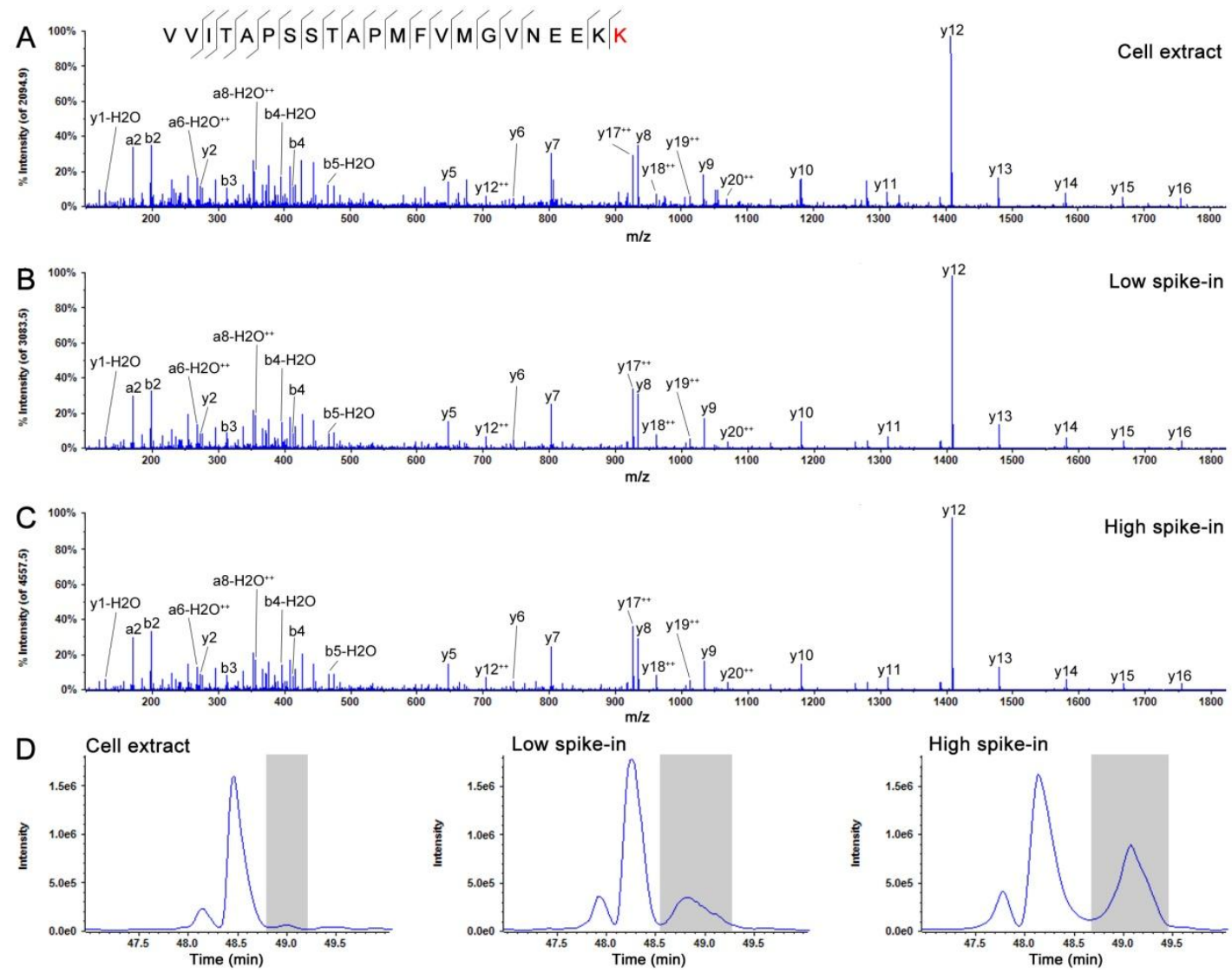

Figure S7. MSMS spectra of the SAP peptide VVITAPSSTAPMFVMGVNEEKK (SAP site in red) detected by PRM analysis in total cell extracts (A), extracts with low spike-in (B) or high spike-in (C) amounts of the synthetic SAP peptide, and corresponding extracted ion chromatograms of the SAP peptide in different samples (D). 